\title{
COLLAPSAR ACCRETION AND THE GAMMA-RAY BURST X-RAY LIGHT CURVE
}

\author{
Christopher C. Lindner ${ }^{1,3}$, Miloš Milosavljević ${ }^{1,2},{\text { Sean M. } \text { Couch }^{1,4} \text {, and Pawan Kumar }}^{1}$ \\ ${ }^{1}$ Department of Astronomy, University of Texas, 1 University Station C1400, Austin, TX 78712, USA \\ 2 Texas Cosmology Center, University of Texas, 1 University Station C1400, Austin, TX 78712, USA \\ Received 2009 August 4; accepted 2010 February 23; published 2010 March 26
}

\begin{abstract}
We present axisymmetric hydrodynamical simulations of the long-term accretion of a rotating gamma-ray burst (GRB) progenitor star, a "collapsar," onto the central compact object, which we take to be a black hole. The simulations were carried out with the adaptive-mesh-refinement code FLASH in two spatial dimensions and with an explicit shear viscosity. The evolution of the central accretion rate exhibits phases reminiscent of the long GRB $\gamma$-ray and X-ray light curve, which lends support to the proposal by Kumar et al. that the luminosity is modulated by the central accretion rate. In the first "prompt" phase, the black hole acquires most of its final mass through supersonic quasiradial accretion occurring at a steady rate of $\sim 0.2 M_{\odot} \mathrm{s}^{-1}$. After a few tens of seconds, an accretion shock sweeps outward through the star. The formation and outward expansion of the accretion shock is accompanied with a sudden and rapid power-law decline in the central accretion rate $\dot{M} \propto t^{-2.8}$, which resembles the $L_{\mathrm{X}} \propto t^{-3}$ decline observed in the X-ray light curves. The collapsed, shock-heated stellar envelope settles into a thick, low-mass equatorial disk embedded within a massive, pressure-supported atmosphere. After a few hundred seconds, the inflow of low angular momentum material in the axial funnel reverses into an outflow from the thick disk. Meanwhile, the rapid decline of the accretion rate slows down, which is potentially suggestive of the "plateau" phase in the X-ray light curve. We complement our adiabatic simulations with an analytical model that takes into account the cooling by neutrino emission and estimate that the duration of the prompt phase can be $\sim 20 \mathrm{~s}$. The model suggests that the steep decline in GRB X-ray light curves is triggered by the circularization of the infalling stellar envelope at radii where the virial temperature is below $10^{10} \mathrm{~K}$, such that neutrino cooling is inefficient and an outward expansion of the accretion shock becomes imminent; GRBs with longer prompt $\gamma$-ray emission should have more slowly rotating envelopes.
\end{abstract}

Key words: accretion, accretion disks - black hole physics - gamma-ray burst: general - stars: winds, outflows supernovae: general

Online-only material: color figures

\section{INTRODUCTION}

Observations of long gamma-ray bursts (GRBs) carried out with the NASA Swift satellite have shown that the $\gamma$-ray prompt emission ceases after about a minute in the observer frame, corresponding to tens of seconds in the rest frame of the progenitor star. The $\gamma$-ray light curve, converted to a fiducial $\mathrm{X}$-ray spectral band, smoothly joins the X-ray light curve, which declines rapidly, $\left(t^{-3}\right.$ or faster) lasting for about $80-300 \mathrm{~s}$ (Tagliaferri et al. 2005; Nousek et al. 2006; O'Brien et al. 2006). The rapid decline is often followed by a phase, from about $\sim 10^{3}$ to $10^{4} \mathrm{~s}$, during which the $\mathrm{X}$-ray flux is roughly constant or declines more slowly with time. The X-ray light curves of some GRBs exhibit "flares" where the flux increases suddenly by a factor of $\lesssim 10^{2}$ and drops precipitously, with the rise and decline associated with the flare occurring on a timescale much shorter than the age of the burst (see, e.g., Burrows et al. 2005; Falcone et al. 2006). Following about $\sim 10^{3}-10^{4} \mathrm{~s}$, a more rapid decline of the luminosity resumes (see, e.g., Zhang et al. 2006, and references therein), and occasionally steepens further at $\sim 10^{4}-10^{5}$ s (e.g., Vaughan et al. 2006).

The goal of the present work is to utilize two-dimensional hydrodynamic simulations to test the hypothesis (Kumar et al. 2008a, 2008b) that this characteristic structure of the X-ray light curve, which was summarized by Zhang et al. (2006), reflects a modulation in the rate of central accretion of a rotating progenitor star onto a black hole or a neutron star,

\footnotetext{
3 NSF Graduate Research Fellow.

4 NASA Earth and Space Science Fellow.
}

as in the collapsar model of GRBs (Woosley 1993; MacFadyen \& Woosley 1999; MacFadyen et al. 2001; Woosley \& Bloom 2006). We do not attempt to explore the implications of the potential presence of a magnetosphere, as in the magnetar model for GRBs (e.g., Duncan \& Thompson 1992; Wheeler et al. 2000; Zhang \& Mészáros 2001; Thompson et al. 2004; Komissarov \& Barkov 2007; Bucciantini et al. 2007, 2009). We will attempt to gain insight in the origin of the steady $\gamma$-ray luminosity (the prompt phase which we will refer to as "Phase 0"), the rapid decline in the X-ray light curve (Phase I in the nomenclature of Zhang et al. 2006), and phase of quasi-steady luminosity or slow decline (Phase II). We will briefly attempt to extrapolate the results of our simulations to the subsequent steeper decline phases (Phases III and IV).

Kumar et al. (2008a, 2008b) obtained the key features of the $\gamma$-ray and X-ray light curve by estimating central accretion rate resulting from the free (i.e., ballistic) infall of a rotating progenitor star. In this picture, the material that has sufficient initial angular momentum to circularize outside of the innermost stable circular orbit (ISCO) of the black hole, forms a disk in the equatorial plane, and subsequently accretes via disk accretion (Narayan et al. 2001). Disk accretion across the ISCO powers an electromagnetic jet that launches from the black hole magnetosphere and pierces the overlying stellar layers. Once outside the star, the electromagnetic and the bulk kinetic energies of the jet dissipate (the precise dissipation mechanism remains poorly understood) and produce the observed emission. Relativistic hydrodynamical simulations (see, e.g., Zhang et al. 2003, 2004; Zhang \& MacFadyen 2006; Morsony et al. 2007; Wang et al. 2008) suggest that a powerful jet might be able 
to pierce the star. A less powerful jet that could be present after few hundred seconds can escape the star only if the axial funnel is kept open by the centrifugal barrier. This seems to be the case in our axisymmetric simulations; threedimensional simulations would be necessary to check that the nonaxisymmetric instabilities do not perturb the rotationally supported fluid along the funnel wall. With this in mind, our interpretation of the observations and the simulations is contingent on the validity of the assumption that the jet can indeed escape.

If the luminosity is then proportional to the central accretion rate, and if the distance of the $\gamma$-ray or X-ray emitting region from the center of the star is assumed to be approximately independent of time on timescales $10-10^{5} \mathrm{~s}$, an accretion model directly translates into a synthetic light curve that can be compared with an observed light curve. Kumar et al. (2008a, 2008b) have shown that with the simplest accretion model involving ballistic infall onto the midplane (assumption also made by Janiuk \& Proga 2008 and Cannizzo \& Gehrels 2009) and subsequent disk accretion, the mapping of the mass accretion history onto the light curve provides a powerful insight into the stratification and angular momentum structure of the progenitor star.

In their ballistic infall model, Kumar et al. (2008a, 2008b) were not able to discriminate between models in which the quasi-steady activity in Phase II arose from disk accretion, or from late-time accretion from an extended stellar envelope. Departures from ballistic infall are expected if the infalling material passes through an accretion shock (see, e.g., MacFadyen \& Woosley 1999; Lee \& Ramirez-Ruiz 2006; Nagataki et al. 2007; Lopez-Camara et al. 2009), or if the disk launches a thermal (MacFadyen 2003a, 2003b; Kohri et al. 2005) or magnetohydrodynamic (e.g., Proga et al. 2003) outflow ("wind") that can interfere with the infall. The existence of the outflow is particularly interesting because of the potential for nucleosynthesis in the free neutron-rich outflow launched from the inner part of the disk (see, e.g., Pruet et al. 2003; Surman et al. 2006; Fujimoto et al. 2007; Nagataki et al. 2007; Maeda \& Tominaga 2009) and because of the potential that the outflow can deplete the accreting stellar envelope and limit the envelope mass that is accreted onto the central black hole.

During the first $\sim 10^{2} \mathrm{~s}$ following the formation of the central black hole when the accretion rate is $\dot{M} \gg 10^{-3} M_{\odot} \mathrm{s}^{-1}$ (the precise condition depends on the black hole spin and shear stress-to-pressure ratio $\alpha$ in the disk), the inner accretion disk cools by neutrino emission and nuclear disintegration and accretes in a radiatively efficient fashion, except for in the very inner, optically thick region (e.g., Popham et al. 1999; Narayan et al. 2001; Di Matteo et al. 2002; Chen \& Beloborodov 2007). Instabilities in the thin disk have been cited as a candidate class of mechanisms that could produce the observed X-ray flares (Perna et al. 2006; Lazzati \& Perna 2007; Lazzati et al. 2008) and could also produce detectable gravitational radiation (Piro \& Pfahl 2007). Our global axisymmetric models are the necessary stepping stone toward the substantially more computationally demanding three-dimensional simulations that will be required to pin down any nonaxisymmetric instabilities in the accreting collapsar (see, e.g., Rockefeller et al. 2006).

We employ two-dimensional unmagnetized hydrodynamic simulations of the collapse, circularization, and accretion of a stellar envelope onto a central point mass, which we assume to be a black hole; relativistic corrections to the gravitational potential are ignored in our simulations since the innermost grid point lies at over 20 Schwarzschild radii in the simulation extending to the smallest radius from the black hole. The torque and dissipation arising from the $R-\phi$ component of the magnetic stress is emulated with a Navier-Stokes term parameterized by an $\alpha$-viscosity prescription. For comparison with the X-ray light curve, we measure the central accretion rate. We track the flow of mass and energy at spherical radii $10^{8} \mathrm{~cm} \lesssim r \lesssim 10^{11} \mathrm{~cm}$ and interpret the results in view of the existing knowledge on radiatively inefficient accretion flows. We observe an outflow and measure the rate at which the accreting stellar envelope is lost to the outflow. The mechanics of postcore-collapse accretion and outflows is key to estimating the final mass of the black hole and the nucleosynthetic composition of the ejected matter (e.g., Zhang et al. 2008, and references therein). The method that we develop here can in future be utilized to estimate the masses of the black holes resulting from the collapse of massive, initially metal-poor "Population III" stars as well as from the collapse of the even more massive, hypothetical "supermassive stars," in the presence of rotation.

In this work, we do not simulate the neutrino-cooled disk, and in the simulations simply impose that the mass that crosses the innermost cylindrical radius of our simulation, $R_{\min }=$ $(0.5-2) \times 10^{8} \mathrm{~cm}$, is instantaneously incorporated inside the black hole and does not provide any further energetic feedback while at radii $R<R_{\min }$. This very rough assumption is bound to fail in general; it is most compatible with the regime in which the transition from efficient to inefficient cooling occurs at $R \gtrsim R_{\min }$. Since the transitional radius for efficient neutrino cooling recedes inward with the increasing stress-to-pressure ratio $\alpha$ for a given accretion rate (Chen \& Beloborodov 2007), the assumption that cooling is efficient within $\lesssim 10^{8} \mathrm{~cm}$ is valid for $\alpha \lesssim 0.01$. We also ignore nuclear disintegration when temperature rises above $\sim(5-10) \times 10^{9} \mathrm{~K}$; in reality, the onset of disintegration allows for some heating via the capture by free nucleons of the neutrinos emitted in the inner disk (Nagataki et al. 2007), which we do not model. However, we do incorporate neutrino cooling in a simple analytical model for the evolution of the accretion shock at the radii that we do not resolve, $\lesssim 5 \times 10^{7} \mathrm{~cm}$. In combination with the simulations, the model provides a theory for the duration of the prompt emission phase observed in the $\gamma$-rays.

Cannizzo \& Gehrels (2009) speculate that a cool, thin disk may form at large radii $\left(R \sim 10^{11} \mathrm{~cm}\right)$ at the onset of Phase II, and attribute the structure of the X-ray light curve to the longterm evolution and slow central accretion of this extended disk. We will see that the formation of the extended thin disk cannot be taken for granted due to the presence of a massive pressuresupported convective atmosphere around the inner disk.

This work is organized as follows. In Section 2, we discuss our numerical algorithm. In Section 3, we present the results our simulations. In Section 4, we present an analytical model for the neutrino-cooled central accretion that we do not resolve in the simulations, and provide a theory for the duration of the prompt accretion phase and the triggering of the steep decline of the $\mathrm{X}$-ray light curve. We also attempt to extrapolate the evolution of the accretion rate beyond the duration of the simulations. Finally, in Section 5, we summarize our conclusions.

\section{NUMERICAL ALGORITHM}

The simulations were carried out with the piecewise-parabolic solver in the adaptive-mesh-refinement code FLASH (Fryxell et al. 2000), version 2.5, in two spatial dimensions using 
cylindrical coordinates $(R, z)$. FLASH does not support angular momentum advection and viscous transport in this regime. In Section 2.1, we describe our implementation of angular momentum transport. In Section 2.2, we discuss our initial model and boundary conditions. In Section 2.3, we provide a test of angular momentum conservation.

\subsection{Angular Momentum Transport}

The specific angular momentum $\ell=R v_{\phi}$, where $v_{\phi}$ is the azimuthal velocity, was treated as a mass scalar quantity that was transported according to (see, e.g., Pringle 1981)

$$
\begin{aligned}
\frac{\partial(\rho \ell)}{\partial t}+ & \frac{1}{R} \frac{\partial\left(R v_{R} \rho \ell\right)}{\partial R}+\frac{\partial\left(v_{z} \rho \ell\right)}{\partial z} \\
& -\frac{1}{R} \frac{\partial}{\partial R}\left[R^{3} v \rho \frac{\partial}{\partial R}\left(\frac{\ell}{R^{2}}\right)\right]=0,
\end{aligned}
$$

where $v$ is a shear viscosity to be specified below. Equation (1), combined with the equation of continuity, is equivalent to the azimuthal axisymmetric Navier-Stokes equation

$$
\frac{\partial v_{\phi}}{\partial t}+v_{R} \frac{\partial v_{\phi}}{\partial R}+\frac{v_{R} v_{\phi}}{R}+v_{z} \frac{\partial v_{\phi}}{\partial z}-\frac{1}{R^{2} \rho} \frac{\partial\left(R^{2} v \rho \sigma\right)}{\partial R}=0
$$

where

$$
\sigma=R \frac{\partial}{\partial R}\left(\frac{v_{\phi}}{R}\right)
$$

is the $R-\phi$ component of the shear tensor. In FLASH, the calculation of the first three terms in Equation (1) is carried out through the mass scalar advection capability; the fourth, parabolic term is included explicitly in the calculation of the radial $\rho \ell$-flux in the code for the diffusion of mass scalars.

The energy dissipated through shear viscosity was accounted for by including the specific heating rate (see, e.g., Landau \& Lifshitz 1959)

$$
\dot{\epsilon}_{\mathrm{visc}}=v\left[R \frac{\partial}{\partial R}\left(\frac{\ell}{R^{2}}\right)\right]^{2}=v \sigma^{2} .
$$

Since we do not simulate the magnetic field of the fluid, we utilize a local definition of the shear viscosity to emulate the magnetic stress arising from the intrinsically nonlocal magnetorotational instability (MRI; Balbus \& Hawley 1998 and references therein). It should be kept in mind, however, that the effects of MRI are in some respects very different from those of the viscous stress. For example, the thick disk surrounding our collapsar black hole is convective; in unmagnetized accretion flows convection transports angular momentum inward, toward the center of rotation (Ryu \& Goodman 1992; Stone \& Balbus 1996; Igumenshchev et al. 2000; Narayan et al. 2000), whereas in magnetized flows, convection can also transport angular momentum outward (Balbus \& Hawley 2002; Igumenshchev 2002; Igumenshchev et al. 2003; Christodoulou et al. 2003). Thus, our results must be interpreted with caution.

Our definition of the local viscous stress emulating the MRI must be valid under rotationally supported, pressure supported, and freely falling conditions. Thompson et al. (2005) suggest that since the wavenumber of the fastest growing MRI mode, which is given by the dispersion relation $v_{\mathrm{A}} k \sim \Omega$ where $v_{\mathrm{A}}$ is the Alfvén velocity and $\Omega=v_{\phi} / R$ is the angular velocity, should in the saturated quasi-state state be about the gas pressure scale height, $k \propto H^{-1}$, the Maxwell $\rho v_{\mathrm{A}}^{2}$ and viscous $v \rho \Omega$ stresses (up to factors in $|d \ln \Omega / d \ln R|$ that we neglect) can be equated if the viscosity is given by

$$
v_{\mathrm{MRI}}=\alpha H^{2} \Omega
$$

where $\alpha$ is a dimensionless parameter. If the pressure scale height is defined locally,

$$
H=|\vec{\nabla} \ln P|^{-1},
$$

the viscosity defined in Equation (5) suffers from divergences at pressure extrema. To alleviate this problem, we define a second viscosity according to the Shakura \& Sunyaev (1973) prescription

$$
v_{\mathrm{SS}}=\alpha \frac{P}{\rho} \Omega^{-1}
$$

Shakura-Sunyaev viscosity overestimates the magnetic stress in stratified hydrostatic atmospheres. We thus set the viscosity in Equations (1) and (4) to equal the harmonic mean of the above two viscosities

$$
v=\frac{2 v_{\mathrm{MRI}} v_{\mathrm{SS}}}{v_{\mathrm{MRI}}+v_{\mathrm{SS}}}
$$

where the pressure gradient in Equation (6) is calculated by the finite differencing of pressure in neighboring fluid cells in the horizontal and vertical directions.

Our choice for the stress-to-total pressure ratio is $\alpha=0.01$, consistent with the ratio of the time-averaged stress to the timeaveraged total pressure in the stratified, radiation-dominated disks in the simulations of Hirose et al. (2009). Hirose et al. found, however, that the fluctuations in the stress and the pressure (total or fluid) are not temporally coincident; this underscores the limitations of our assumed direct proportionality of the viscous stress with the total pressure. In the limits $v_{\text {MRI }} \gg v_{\text {SS }}$ or $v_{\text {MRI }} \ll v_{\text {SS }}$, the effective value of the viscosity parameter implied by Equation (8) is twice the nominal value, $\alpha_{\text {eff }} \approx 0.02$.

Because FLASH employs an explicit method for the diffusion of mass scalars, numerical stability of the above viscous transport prescription places a stringent upper limit on the time step

$$
\Delta t<\frac{\Delta R^{2}}{2 v}
$$

where $\Delta R$ is the grid resolution. For $\alpha \gg 0.01$, the viscous time step in our simulations becomes prohibitively shorter than the Courant time step. In our test simulations with a $\gamma$-law equation of state (EOS), we find that while not implying an outright instability, a choice of $\Delta t$ that saturates the limit in Equation (9) results in weak stationary staggered perturbations in the fluid variables. We ignore this complication and allow our time step to be set by the limit in Equation (9) of the cell with the smallest viscous diffusion time across the cell.

The centrifugal force is included in the calculation of the gravitational acceleration via

$$
\vec{a}_{\text {grav }}=-\frac{G M_{\mathrm{BH}}}{r^{3}} \vec{r}-\vec{\nabla} \Phi_{\mathrm{fl}}+\frac{\ell^{2}}{R^{4}} \vec{R}
$$

where $r=\left(R^{2}+z^{2}\right)^{1 / 2}, M_{\mathrm{BH}}$ is the mass of the central compact object which we take to be a black hole, and $\Phi_{\mathrm{fl}}(r)$ is the spherically symmetric component of the gravitational potential 
Table 1

Summary of Simulation Parameters and Key Measurements

\begin{tabular}{|c|c|c|c|c|c|c|c|c|c|}
\hline Run Number & $R_{\min }(\mathrm{cm})^{\mathrm{a}}$ & $(R, z)_{\max }(\mathrm{cm})^{\mathrm{b}}$ & $\Delta(R, z)_{\min }(\mathrm{cm})^{\mathrm{c}}$ & $M_{\mathrm{BH}, \text { init }}\left(M_{\odot}\right)^{\mathrm{d}}$ & $t_{\max }(\mathrm{s})^{\mathrm{e}}$ & $\alpha^{\mathrm{f}}$ & $t_{\text {decl }}(\mathrm{s})^{\mathrm{g}}$ & $M_{\mathrm{BH}, \mathrm{decl}}\left(M_{\odot}\right)^{\mathrm{h}}$ & $(d \ln \dot{M} / d \ln t)_{\mathrm{decl}}$ \\
\hline 1 & $5 \times 10^{7}$ & $5 \times 10^{11}$ & $1.9 \times 10^{6}$ & 0.51 & $10^{2}$ & 0.01 & 37 & 7.35 & -2.8 \\
\hline 2 & $10^{8}$ & $10^{11}$ & $6.1 \times 10^{6}$ & 1.26 & $10^{3}$ & 0.01 & 47 & 8.97 & $-2.7^{\mathrm{j}}$ \\
\hline 3 & $2 \times 10^{8}$ & $5 \times 10^{11}$ & $7.6 \times 10^{6}$ & 2.05 & $2 \times 10^{3}$ & 0.01 & 52 & 10.44 & $-2.3^{k}$ \\
\hline
\end{tabular}

Notes.

a The minimum cylindrical radius.

b The maximum cylindrical radius and absolute vertical latitude.

c The minimum resolution element size.

d Initial black hole mass.

e Duration of the simulation.

f The viscous stress parameter (see Section 2.1).

$\mathrm{g}$ Time of the beginning of the steep decline of the accretion rate.

h Black hole mass at the beginning of the steep decline of the accretion rate.

${ }^{\mathrm{i}}$ Logarithmic slope of the decline of the accretion rate.

j For $50 \mathrm{~s} \leqslant t \leqslant 500 \mathrm{~s}$.

${ }^{\mathrm{k}}$ For $52 \mathrm{~s} \leqslant t \leqslant 200 \mathrm{~s}$.

of the fluid within the computational grid. The latter is calculated with the multipole Poisson solver in FLASH via

$$
\begin{aligned}
\Phi_{\mathrm{fl}}(r)=- & \int_{r<r^{\prime}} \frac{G \rho\left(R^{\prime}, z^{\prime}\right)}{r^{\prime}} 2 \pi R^{\prime} d R^{\prime} d z^{\prime} \\
& -\int_{r>r^{\prime}} \frac{G \rho\left(R^{\prime}, z^{\prime}\right)}{r} 2 \pi R^{\prime} d R^{\prime} d z^{\prime},
\end{aligned}
$$

where $r^{\prime} \equiv\left(R^{\prime 2}+z^{\prime 2}\right)^{1 / 2}$. While the distribution of mass at small radii becomes highly flattened, the gravitational potential there is dominated by the central point mass, and thus, the neglect of all but the spherical multipole is an acceptable approximation. At the radii and densities that we resolve in the simulations, relativistic effects are weak; we thus treat the gravitational potential as Newtonian. In Section 2.3, we present a test of the angular momentum transport code.

\subsection{Initial Model and Boundary Conditions}

The initial model is the rotating $\approx 14 M_{\odot}$ Wolf-Rayet star 16TI of Woosley \& Heger (2006), evolved to pre-core-collapse from a $16 M_{\odot}$ main-sequence progenitor. ${ }^{5}$ To prepare the model 16 TI, Woosley \& Heger assumed that the rapidly rotating progenitor, which is near breakup at its surface at $r \approx 4 \times$ $10^{10} \mathrm{~cm}$, had low metallicity, $0.01 Z_{\odot}$, on the main sequence and became a WR star shortly after central $\mathrm{H}$ depletion, which implied an unusually small amount of mass loss. For illustration, the specific angular momentum at the three-quarters mass radius was $\ell_{3 / 4} \sim 8 \times 10^{17} \mathrm{~cm}^{2} \mathrm{~s}^{-1}$, implying circularization around a $10 M_{\odot}$ black hole at $R \sim 5 \times 10^{8} \mathrm{~cm}$. The circularization radii of the outermost layers of the star are in the range $10^{9}-10^{10} \mathrm{~cm}$. Woosley \& Heger provide a radius-dependent angular momentum profile $\ell(r)$; we endowed this with a dependence on the polar angle $\theta \equiv \cos ^{-1}(z / r)$ via

$$
\ell(r, \theta)=\ell(r) \sin ^{2}(\theta),
$$

such that the star rotates rigidly on spherical shells. In Figure 4, we show the density, temperature, pressure, specific angular momentum, and mean atomic number of the initial model (black line).

\footnotetext{
5 Lopez-Camara et al. (2009) carried out smoothed particle hydrodynamics (SPH) simulations of neutrino-cooled accretion during the first $0.5 \mathrm{~s}$ of the collapse and Morsony et al. (2007) simulated the propagation of a relativistic jet using the same model star.
}

We placed the center of the star at the origin, $(R, z)=$ $(0,0)$. Pseudo-logarithmic gridding was achieved by capping the adaptive resolution at radius $r$ with $\Delta R, \Delta z>\frac{1}{8} \eta r$, where we choose $\eta=0.05$; this prevents use of excessive resolution far from the center of the star. Beyond the outer edge of the star at $r_{\text {star }}=4 \times 10^{10} \mathrm{~cm}$, we placed a cold $\left(10^{4} \mathrm{~K}\right)$ low-density stellar-wind-like medium with density profile $\rho(r)=3 \times 10^{-7}\left(r / r_{\text {star }}\right)^{-2} \mathrm{~g} \mathrm{~cm}^{-3}$. Since the model 16TI of Woosley \& Heger (2006) was not constructed to be in hydrostatic equilibrium in the presence or rotation, we ignored rotation and set the initial density distribution to be spherically symmetric at the beginning of the simulations. This is a poor approximation in the very outer layers of the star, as is evident from the ellipsoidal distortion that sets in at the beginning of the simulation.

For the EOS we chose the Helmholtz EOS provided with the FLASH distribution (Timmes \& Swesty 2000), which contains contributions to pressure and internal energy from radiation, ions, electrons, positrons, and Coulomb effects. We passively advected the abundances of seven nuclear species represented in the model including ${ }^{4} \mathrm{He},{ }^{12} \mathrm{C},{ }^{16} \mathrm{O},{ }^{20} \mathrm{Ne},{ }^{24} \mathrm{Mg},{ }^{28} \mathrm{Si}$, and ${ }^{56} \mathrm{Fe}$. The local nuclear composition was passed to the Helmholtz EOS as input. We do not simulate nuclear reactions, nuclear disintegration, and neutrino emission and absorption. These processes are certainly important in the hot inner accretion disk around the collapsar black hole, but since we simulate only the outer, cooler disk with temperatures $T<10^{10} \mathrm{~K}$, the neglect of nuclear and neutrino processes is a reasonable approximation.

The simulation was carried out in the annular cylindrical domain $R_{\min }<R<R_{\max }$ and $-z_{\max }<z<z_{\max }$. Because the impact of time-step limitations (Equation (9)) on the computational cost, and to avoid dealing with the fluid hot enough to be susceptible to efficient neutrino cooling, the smallest inner radius $R_{\min }$ that we could simulate was $R_{\min } \sim 5 \times 10^{7} \mathrm{~cm}$. We placed the outer boundaries well outside the star $R_{\max }=z_{\max }=(1-5) \times 10^{11} \mathrm{~cm}$. In Table 1, we summarize the main parameters of our simulations, and also present some of the key measurements, defined in Section 3, characterizing the outcome of each simulation. Each simulation was run for $\sim 10^{6}$ hydrodynamic time steps and consumed $\sim 20,000$ CPU hours on the Texas Advanced Computing Center's clusters Lonestar and Ranger.

The boundary condition at $R_{\min }$ was unidirectional outflow that allowed free flow from larger to smaller radii (off the grid) 
and disallowed flow from smaller to larger radii (onto the grid) by imposing a reflecting boundary condition at $R_{\min }$ whenever $v_{R}$ was positive in the leftmost grid cell. We imposed the torquefree boundary condition ${ }^{6}$ via

$$
\frac{\partial}{\partial R}\left(\frac{\ell}{R^{2}}\right)_{R=R_{\min }}=0 .
$$

As in other Eulerian codes, the boundary conditions in FLASH are imposed by assigning values to fluid variables in rows of "guard" cells just outside the boundary of the simulated domain. At any given value of $z$ on the computational grid, let $R_{1 / 2}$ denote the leftmost cell within the simulated domain, and let $R_{g}$ where $g=\left(-\frac{7}{2},-\frac{5}{2},-\frac{3}{2},-\frac{1}{2}\right)$ be the four guard cells to the left of $R_{1 / 2}$ such that the grid separation corresponds to $\Delta g=1$. The torque-free boundary condition, if assumed to apply for $R \leqslant R_{\min }$, implies $\ell_{g} / R_{g}^{2}=\ell_{1 / 2} / R_{1 / 2}^{2}$. We fixed the guard-cell velocity perpendicular to the left vertical boundary to $v_{R, g}=-\left|v_{R, 1 / 2}\right| R_{1 / 2} / R_{g}$, which, with the assumption of uniform density $\rho_{g}=\rho_{1 / 2}$, ensures mass continuity in the guard cells and the vanishing of the mass flux across $R=R_{\min }$ if $v_{R, 1 / 2}>0$. All other fluid variables $X$ were simply copied into the guard cells, $X_{g}=X_{1 / 2}$, and were subsequently rendered thermodynamically consistent. This simple prescription approximates free inflow (toward smaller $R$ ) across $R_{\min }$, but of course, the guard-cell values violate energy and momentum conservation at $R<R_{\min }$.

The mass of the black hole $M_{\mathrm{BH}}$ was initialized with the stellar mass initially located outside the grid, at $R<R_{\min }$. The black hole mass was evolved by summing mass flux crossing the boundary at $R=R_{\min }$,

$$
\frac{d M_{\mathrm{BH}}}{d t}=-2 \pi R_{\min } \int_{-z_{\max }}^{z_{\max }} v_{R}\left(R_{\min }, z\right) \rho\left(R_{\min }, z\right) d z .
$$

\subsection{Test of the Code}

To test our implementation of angular momentum transport, we performed a one-dimensional $(\partial / \partial z=0)$ simulation of an initially uniform temperature and density fluid with the $\gamma=\frac{5}{3}$ EOS orbiting in a Keplerian potential. We use a uniform radial grid with $R_{\max }=100 R_{\min }$ and grid spacing $\Delta R=0.06 R_{\min }$. The initial temperature was chosen such that the sound speed was about $16 \%$ of the Keplerian velocity at the inner radial boundary, and 1.6 times the Keplerian velocity at the outer radial boundary. The time step was limited by $\Delta t \leqslant \frac{1}{4} \Delta R^{2} / v$, which is a factor of 2 more stringent than the stability condition in Equation (9). We found that reducing the time step to a half of the value required for stability substantially reduces, but does not entirely eliminate, the noise in the error estimator that we are about to discuss. We evaluate the nonzero terms in Equation (1) directly from the numerical data. Let the $\tau_{1}, \tau_{2}$, and $\tau_{3}$ denote the first, second, and fourth term in Equation (1)

$$
\begin{aligned}
\tau_{1} & \equiv \frac{\partial(\rho \ell)}{\partial t}, \\
\tau_{2} & \equiv \frac{1}{R} \frac{\partial\left(R v_{R} \rho \ell\right)}{\partial R}, \\
\tau_{3} & \equiv-\frac{1}{R} \frac{\partial}{\partial R}\left[R^{3} v \rho \frac{\partial}{\partial R}\left(\frac{\ell}{R^{2}}\right)\right] .
\end{aligned}
$$

\footnotetext{
6 A motivation of the torque-free boundary condition can be found in Zimmerman et al. (2005).
}

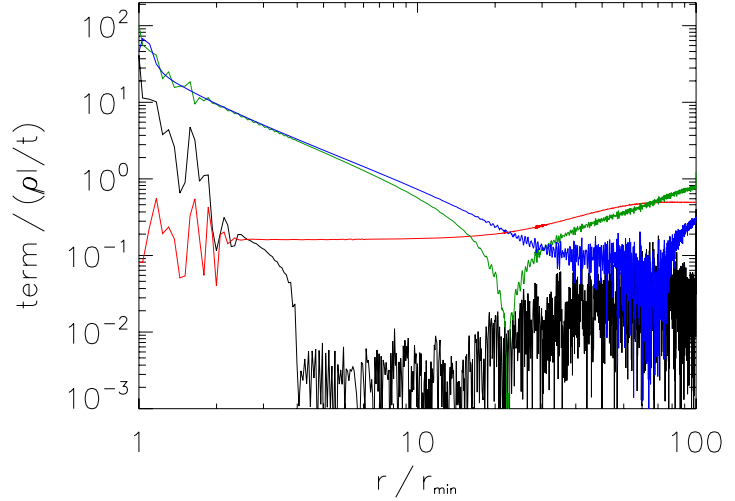

Figure 1. Test of angular momentum transport, showing the magnitude of the Eulerian time derivative ( $\tau_{1} ;$ red curve), advection ( $\tau_{2} ;$ green curve), and viscous transport ( $\tau_{3}$; blue curve) terms in the one-dimensional $(\partial / \partial z=0)$ version of the angular momentum conservation relation in Equation (1). The terms are expressed in the units of $\rho \ell / t$. We also show the sum $\left|\tau_{1}+\tau_{2}+\tau_{3}\right|$ (black curve), which should be much smaller than the largest of the three terms. The violation of angular momentum conservation near the left radial boundary is associated with the conservation-violating nature of the torque-free boundary condition that we have imposed.

\section{Correct angular momentum transport requires}

$$
\left|\tau_{1}+\tau_{2}+\tau_{3}\right| \ll \max \left(\left|\tau_{1}\right|,\left|\tau_{2}\right|,\left|\tau_{3}\right|\right) .
$$

In Figure 1, we plot $\left|\tau_{1}\right|,\left|\tau_{2}\right|,\left|\tau_{3}\right|$, and $\left|\tau_{1}+\tau_{2}+\tau_{3}\right|$ over the entire range of radii after $\sim 1000$ Keplerian orbital periods at $R_{\min }$, which corresponds to $\sim 1$ orbital period at $R_{\max }$. Radial derivatives were computed by three-point Lagrangian interpolation with the IDL routine DERIV. In computing the derivative at inner boundary, we included guard cells in manner equivalent with the boundary condition prescription used in our two-dimensional simulations, as described in Section 2.2. Some violation of the transport Equation (1) is expected at the two leftmost grid cells at $R \approx(1.03,1.09) \times R_{\min }$ because the torque-free boundary condition does not conserve angular momentum. Apart from the leftmost cells, the angular momentum is conserved at the $10 \%$ level or better at all radii. The spatial derivative in the viscous transport term $\left(\tau_{3}\right)$ is partially responsible for the noise evident at large radii. The noise at small radii seems to be correlated with the viscous time-step limiter, which suggests that it is related to the explicit nature of our viscous diffusion scheme.

\section{RESULTS}

Since the evolution of the central accretion rate seems to fall into three distinct phases (see Figure 2) that appear to correspond to the phases identified in the GRB $\gamma$-ray and X-ray light curve (e.g., Zhang et al. 2006), we divide our description of the results of the simulations into three parts. In Section 3.1, we describe Phase 0 that concludes with the appearance of an accretion shock. We also discuss the limitations of our method in this regime, and set the stage for an analytic model that we develop further below in Section 4.1 to take into account the physics left out the our simulations. In Section 3.2, we describe Phase I that is characterized by a steep, power-law decline of the central accretion rate and a rapid hydrodynamic readjustment of the accreting stellar envelope. In Section 3.3, we describe Phase II, in which the central accretion rate steadies. The corresponding "plateau" phase in GRB X-ray light curves eventually ends and gives way to a renewed steeper decline. Because of computational cost limitations, we do not extend our 


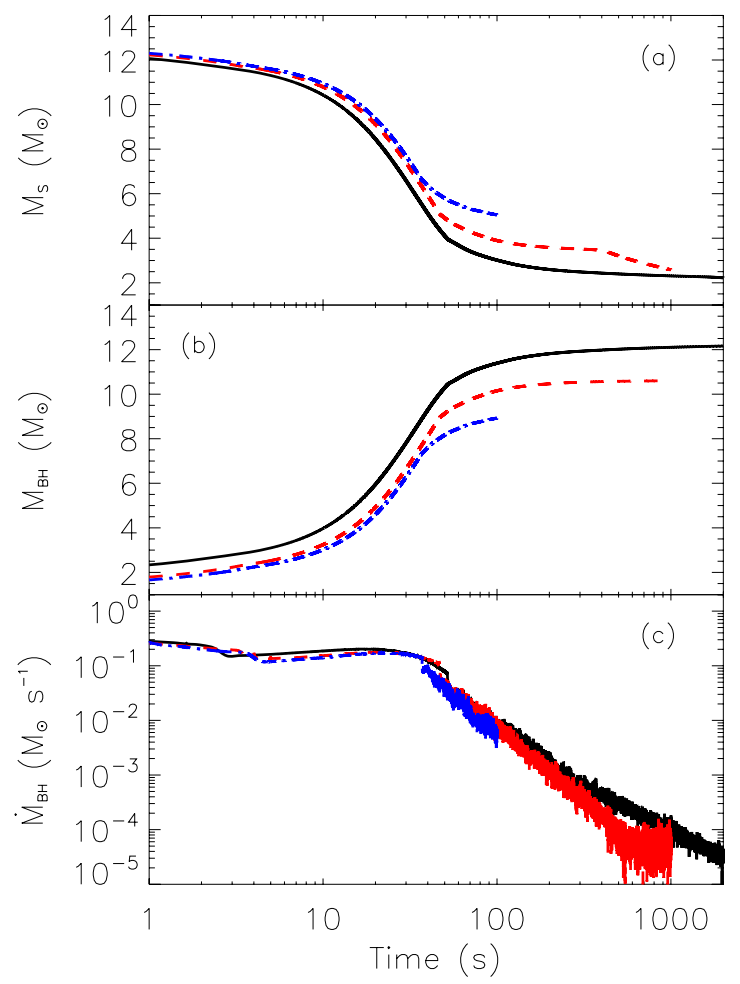

Figure 2. (a) Stellar mass that remains in the simulation as a function of time in Run 1 (blue, dot-dashed line), Run 2 (red, dashed line), and Run 3 (black, solid line). The drop at $t \approx 400 \mathrm{~s}$ in Run 2 is an artifact of fluid escape from the box through the boundaries at $R=R_{\max }$ and $z= \pm z_{\max }$. (b) Mass of the black hole. (c) The rate at which fluid mass accretes across the boundary at $R=R_{\min }$ and is added to the mass of the black hole. The sharp drop at $t \approx 37-52$ s coincides with the formation of the accretion shock and the onset of convection in the rotationally and hydrostatically supported fluid. The flattening at $t \sim 500 \mathrm{~s}$ in Run 2 coincides with the cessation of the accretion of low angular momentum fluid through the axial funnel. The power-law accretion rate decline in Run 3 exhibits a shallowing of the logarithmic slope at $t \sim 200 \mathrm{~s}$.

(A color version of this figure is available in the online journal.)

runs to $\sim 10^{4} \mathrm{~s}$, where, based on the observed light curves, one would expect the renewed steeper decline to occur, but further below, in Section 4.2, we briefly speculate about the long-term evolution of the light curve.

\subsection{Phase 0: Quasiradial Accretion}

At the radii $R>R_{\min }$ that we resolve in the simulations, the stellar collapse proceeds quasiradially for a number of seconds until an accretion shock appears at the innermost simulated point at $(R, z)=\left(R_{\min }, 0\right)$. The appearance of an accretion shock coincides with the emergence of a rotationally supported flow in the zones at the smallest radii. In Figure 3, left panel, we show the density distribution, temperature contours, and velocity field during the quasiradial accretion phase, at $t=37 \mathrm{~s}$, just prior to the formation of the accretion shock, in the simulation with $R_{\min }=5 \times 10^{7} \mathrm{~cm}$ (Run 1 ; see Table 1 ). The maximum density and temperature in this snapshot are $2.6 \times 10^{7} \mathrm{~g} \mathrm{~cm}^{-3}$ and $T=7.5 \times 10^{9} \mathrm{~K}$. Since the simulation does not allow for nuclear disintegration, the temperature in the innermost cells in the simulation is an overestimate.

The existence of a quasiradial accretion phase and the late formation of an accretion shock are clearly artifacts of the choice not to simulate the innermost $5 \times 10^{7} \mathrm{~cm}$ from the central axis. This innermost resolved radius is still $\sim 100$ times larger than the gravitational radius of the nascent black hole. Simulations that resolved the innermost radii at or near the
ISCO (MacFadyen \& Woosley 1999; Proga et al. 2003; Lee \& Ramirez-Ruiz 2006; Nagataki et al. 2007; Barkov \& Komissarov 2008; Komissarov \& Barkov 2009; Lopez-Camara et al. 2009), but were run much shorter than ours, saw accretion shock formation much earlier, during the first second of the collapse. Some of the material falling quasiradially during the initial phase has enough angular momentum to circularize at radii that we do not resolve, but that are still larger than the ISCO. Indeed, our accretion shock forms earlier in runs with smaller $R_{\min }$ (see Table 1 and Figure 2), consistent with the observation that circularization triggers shock formation. Therefore, in general, the accretion shock forms when the orbital pericenter of the material crossing the equatorial plane becomes larger than the ISCO, or the innermost resolved radius $R_{\min }$ in the simulations in which the ISCO is unresolved.

In Section 4.1, we will analyze differences between the inner accretion flow in our adiabatic simulations and that in the realistic GRBs progenitors, and suggest that the steep decline of the accretion rate in realistic GRB progenitors is triggered by the onset of circularization of the infalling stellar material at radii where the post-accretion-shock temperature is too low to allow for efficient cooling by neutrino emission. We will conclude that the decline seems to be associated with the onset of outward expansion of the accretion shock. The outward expansion is distinct from and could occur much later than the first occurrence of the shock. In Section 4.1, we will present a crude analytic model in which we estimate that the triggering of the steep decline should occur at $t_{\mathrm{decl}} \sim 20 \mathrm{~s}$ in stars with density and angular momentum stratification as in 16TI. This estimate is somewhat shorter than the shortest interval $t_{\mathrm{decl}} \sim 37 \mathrm{~s}$ observed in the highest-resolution simulation, Run 1.

\subsection{Phase I: Funnel and Thick Disk Accretion}

At $t \sim 37-52 \mathrm{~s}$ where the shortest timescale corresponds to the simulations that resolve the smallest radii, an accretion shock forms along the equator near the inner boundary (Figure 3 ) and travels outward. Figure 4 shows equatorial profiles of the density, temperature, pressure, specific angular momentum, mean atomic mass, and vertical pressure scale height, at times $t=(38,100,1000) \mathrm{s}$. The shock travels from $r \approx 2 \times$ $10^{8} \mathrm{~cm}$ at $t=38 \mathrm{~s}$ to $r \approx 10^{10} \mathrm{~cm}$ at $t=100 \mathrm{~cm}$ and escapes the star in the next $\sim 100$ s. In the same period, the shock velocity increases from $\sim 5 \times 10^{7} \mathrm{~cm} \mathrm{~s}^{-1}$ to $\sim 1.5 \times$ $10^{8} \mathrm{~cm} \mathrm{~s}^{-1}$. The shocked fluid at polar angles $|\theta-\pi / 2| \lesssim 75^{\circ}$ is rotationally supported, but the large equatorial vertical pressure scale height $h \equiv\left[r^{2} P /(\rho d \Phi / d r)\right]^{1 / 2} \sim(0.4-0.5) r$, where $\Phi(r)$ is the gravitational potential, indicates that this is a thick disk. Figure 5, left panel, shows that the isodensity contours of this rotationally supported fluid are roughly circular; the vertical and the cylindrically radial pressure scale heights are comparable. The shocked fluid is turbulent and apparently convective (in two spatial dimensions, long-lived vortices form in the shock; the persistence of the vortices is an artifact of the assumed axisymmetry). The near-uniformity of specific angular momentum and mean mass is indicative of rapid mixing. The maximum temperature in the post-shock fluid is $9.3 \times 10^{9} \mathrm{~K}$; this is a temperature at which the nuclear and neutrino physics that we ignore is marginally important; our neglect of disintegration cooling implies an overestimate of the temperature in the inner thick disk, $r \lesssim 10^{8} \mathrm{~cm}$. The shocked fluid in the $|\theta-\pi / 2| \gtrsim 75^{\circ}$ cone around the vertical axis continues to infall supersonically.

Figure 6 shows the quantity $p / \rho^{\gamma}$, which is approximately related to the specific entropy, where $\gamma \equiv(d \ln p / d \ln \rho)_{s=\text { const }}$ 

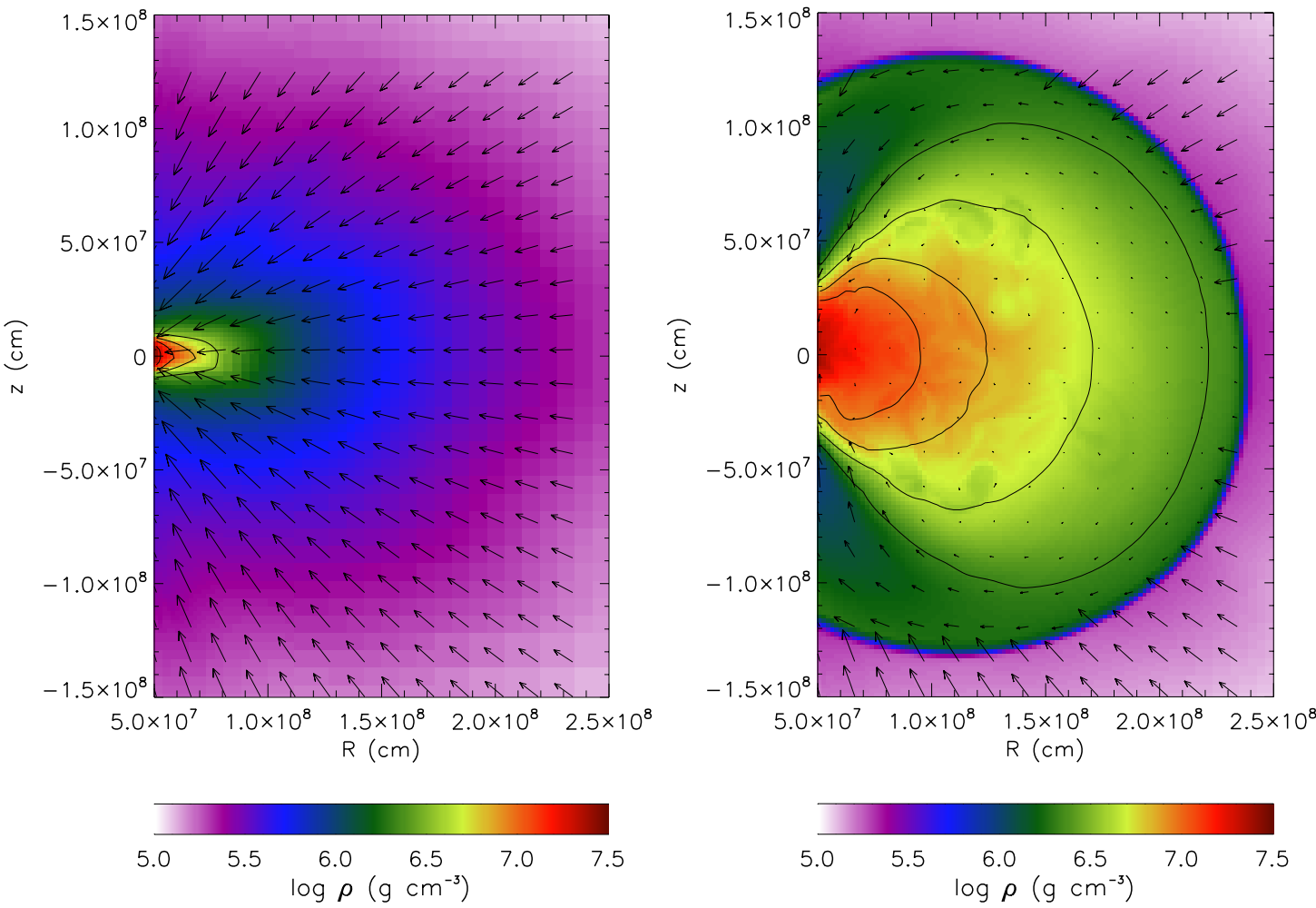

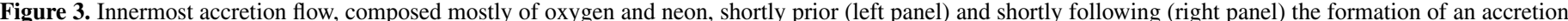

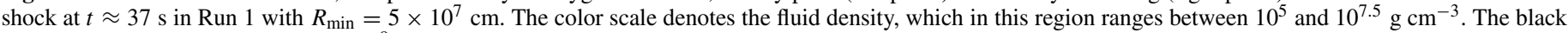

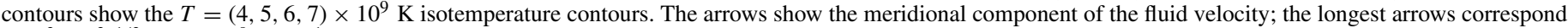
to $\left(v_{R}^{2}+v_{z}^{2}\right)^{1 / 2}=5 \times 10^{9} \mathrm{~cm} \mathrm{~s}^{-1}$ and Mach numbers $\sim 10$. Following shock formation, supersonic inflow resumes along the axial funnel.

at $t=70 \mathrm{~s}$ and $t=100 \mathrm{~s}$ in the highest-resolution run, Run 1. Entropy appears to be generated throughout the thick disk. The high-entropy fluid exhibits a flow morphology suggestive of a "disk wind." The strongest outflow tracked by the highest entropy fluid is along the interface of the turbulent thick equatorial disk and the supersonic axial inflow. Prior to the cessation of the axial inflow, the wind streamlines do not terminate at infinity, but rather bend back toward the equatorial plane, suggesting a closed meridional circulation pattern that transports the energy generated in the thick disk. Ohsuga et al. (2005) and Lee \& Ramirez-Ruiz (2006) have previously observed such a largescale circulation pattern in their simulations. The high-entropy fluid appears to accumulate at the interface of the thick, rotationally supported disk and the pressure-supported atmosphere, and to mix convectively in the atmosphere.

The appearance of the accretion shock is accompanied by a sudden rapid power-law decline of the central accretion rate. The times of shock formation and the onset of decline in different simulations are provided in Table 1. Evolution of the black hole mass, the residual stellar fluid mass, and the accretion rate, is shown in Figure 2. The steep decline of the accretion rate resembles the rapid decline ubiquitous in the observed GRB X-ray light curves. In the simulations, the decline starts at $\sim 37-52 \mathrm{~s}$ and lasts until $\sim 200-500 \mathrm{~s}$. The logarithmic derivative of the central accretion rate during the decline is $d \ln \dot{M} / d \ln t \approx-2.3-(-2.8)$, with the steepest decline corresponding to Run 1 , the simulation that resolves the smallest radii.

There does not seem to be a single explanation for the steepness of the decline of the accretion rate. We have been able to identify three processes that seem to contribute. We focus on Run 2, the run with the highest central resolution that we have run long enough to witness the end of the decline.

First, the Eulerian density within the thick disk decreases by a factor of $\sim 50-100$ from $t=50 \mathrm{~s}$ to $500 \mathrm{~s}$. The density drop occurs concurrently with the accretion shock expansion, and may be associated with the draining of the inner disk into the black hole and with a simultaneous readjustment of the pressure-supported atmosphere of the disk toward nearadiabatic stratification in the presence of convection or largescale circulation. This decline in disk density can explain $d \ln \dot{M} / d \ln t \approx-2$ but perhaps not steeper.

Second, there is a very gradual decrease, by a factor of $\lesssim 2$, of the vertical pressure scale height of the rotationally supported disk during the period of the steep decline. The decrease can be seen in a comparison of the left panel of Figure 5, showing the density distribution at $t=100 \mathrm{~s}$, with the right panel of the same figure, showing the density at $t=1000 \mathrm{~s}$. Since in the rotationally supported flow the viscosity is proportional to the square of the scale height, the scale height decrease implies a factor of $\lesssim 4$ decrease of the viscosity $v$, and with it also of the disk accretion rate $\dot{M}_{\text {disk }}$. Consistent with the disk scale height decrease, the midplane temperature of the disk decreases gradually and steadily. For example, in Run 2 at the innermost resolved radius of $10^{8} \mathrm{~cm}$, the temperature drops from $5 \times 10^{9} \mathrm{~K}$ at the onset of circularization to $2 \times 10^{9} \mathrm{~K}$ at the end of Phase I.

Third, there is a rapid decline of the rate at which the low-angular-momentum fluid accretes through the axial funnel. Funnel accretion dominates the net accretion rate immediately following accretion shock formation but then drops to zero at the end of the steep decline at $t \sim 500 \mathrm{~s}$ when the funnel inflow reverses into an outflow. Our simulations may overestimate the 

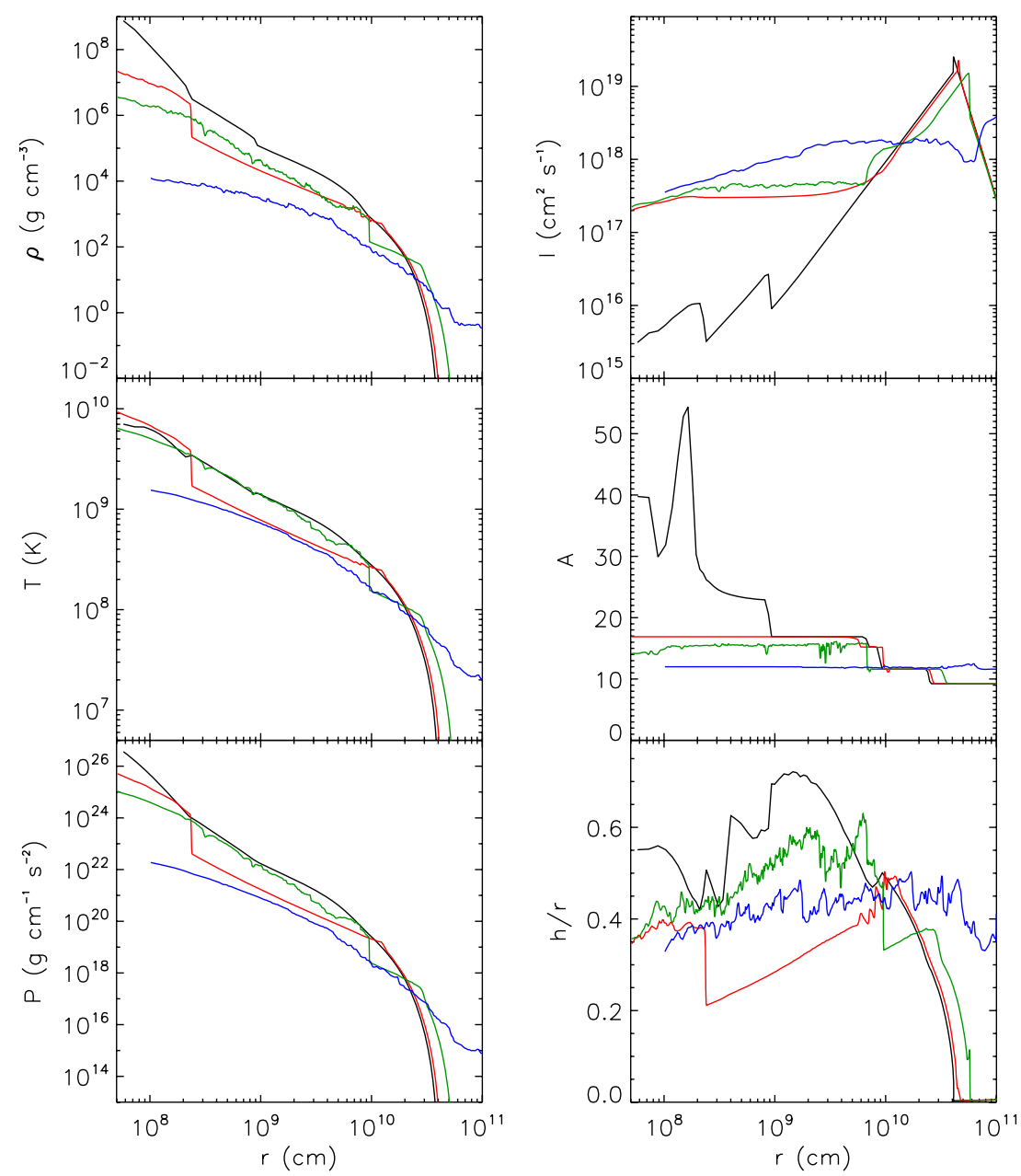

Figure 4. Density $\rho$, temperature $T$, pressure $P$, specific angular momentum $\ell$, mean atomic mass $\bar{A}$, and the vertical pressure scale height-to-radius ratio $h / r$ in the equatorial $(z=0)$ plane. Shown is the initial model (black line), in Run 1 shortly following accretion shock formation at $t=38 \mathrm{~s}$ (red line), in Run 1 at $t=100 \mathrm{~s}$ (green line), and in Run 2 at $t=1,000 \mathrm{~s}$ (blue line). As usual, the mean atomic mass was calculated via $\bar{A}^{-1}=\sum_{i} A_{i}^{-1} X_{i}$, where $A_{i}$ and $X_{i}$ are the atomic masses and mass fractions of the seven nuclear species. The pressure scale height was estimated via $h=\left[r^{2} P /(\rho d \Phi / d r)\right]^{1 / 2}$, where $\Phi$ is the gravitational potential. The outgoing shock is visible at $r \approx 2.3 \times 10^{8} \mathrm{~cm}$ at $t=38 \mathrm{~s}$ and at $r \approx 9 \times 10^{9} \mathrm{~s}$ at $t=100 \mathrm{~s}$. The profiles can be compared with the corresponding profiles in Popham et al. (1999), MacFadyen \& Woosley (1999), and Chen \& Beloborodov (2007).

funnel accretion rate if the funnel material is additionally heated by a narrow relativistic axial jet, presumably launched from the black hole magnetosphere and responsible for the $\gamma$-ray and $\mathrm{X}$-ray emission, that we do not simulate, but which must pierce the funnel region (see, e.g., Ramirez-Ruiz et al. 2002; Zhang et al. 2003, 2004; Zhang \& MacFadyen 2006; Morsony et al. 2007; Wang et al. 2008). The effect of the heating of the funnel fluid by the relativistic jet might be to further steepen the decline of the accretion rate. Further magnetic outflow could develop from the corona of the inner accretion disk, which could shut off funnel accretion more effectively than the outflow driven thermally by the resistive (or, in our approximation, viscous) dissipation in the disk (Proga \& Begelman 2003; Proga et al. 2003). These effects could clearly make the central accretion rate decline, which is already rapid in our simulations, become even more rapid.

One also expects that the accretion rate decline is accompanied by an inward recession of the boundary separating the radiatively efficient, neutrino-dominated accretion flow (NDAF) and the radiatively inefficient, advection or convection dominated accretion flow (RIAF; see, e.g., Chen \& Beloborodov 2007). The evolution of an NDAF into an RIAF at radii $r \lesssim 10^{8} \mathrm{~cm}$ over the course of a few hundred seconds is a process that may further accelerate the accretion rate decline in real GRB progenitors. The physics of the transition from NDAF to RIAF and the onset of the outward propagation of the accretion shock are closely linked - both are controlled by neutrino cooling. We will argue in Section 4.1 that the two operating together, starting at about the same time, is the most likely reason for the rather steep decline of the accretion rate between several tens of seconds and several hundred seconds.

The rapid decline of the central accretion rate in our simulations is distinct in origin from the less rapid decline seen in the simulations of MacFadyen et al. (2001). MacFadyen et al. simulated the fallback of the stellar envelope following the failure of the shock wave resulting from the core bounce to unbind the star. Placing their inner numerical boundary at $r_{\min , \mathrm{MHW}}=10^{9} \mathrm{~cm}$, they found that the radial fallback rate through the inner boundary declines at the rate $\dot{M}\left(r_{\text {min,MHW }}\right) \propto t^{-5 / 3}$. Since the boundary was place outside the radii of the infalling envelope encounters the centrifugal barrier, MacFadyen et al. did not simulate the accretion disk and thus did not observe the formation and outward propagation of the accretion shock. In our simulations, the accretion shock is aided by the viscous energy deposition in the rotationally supported disk. The post-circularization shock seems to be responsible for the much more rapid central 

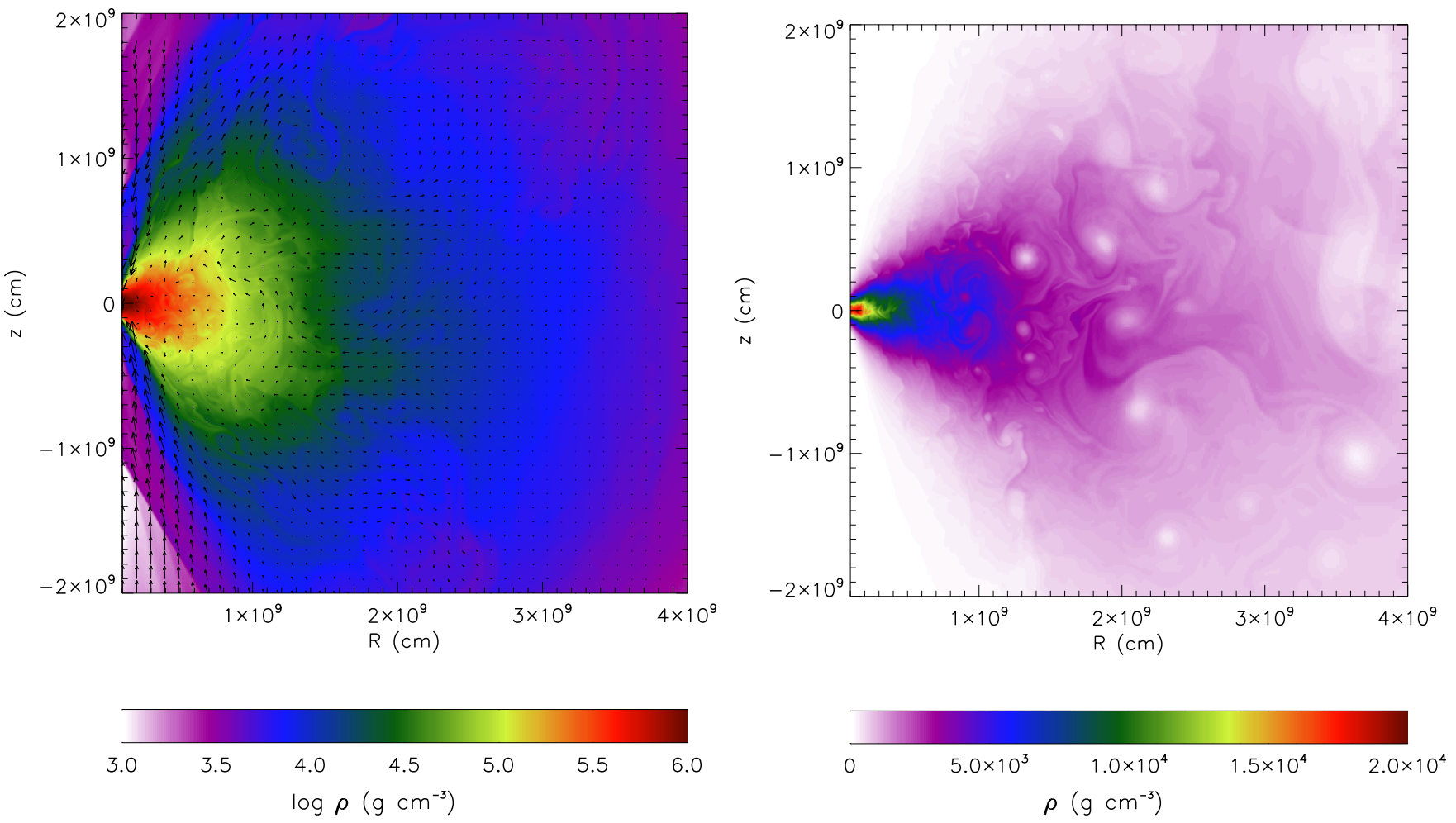

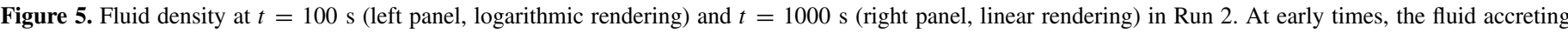
supersonically through the axial funnel traverses multiple weak standing accretion shocks before it joins the disk or passes the boundary at $R=R_{\text {min }}$.
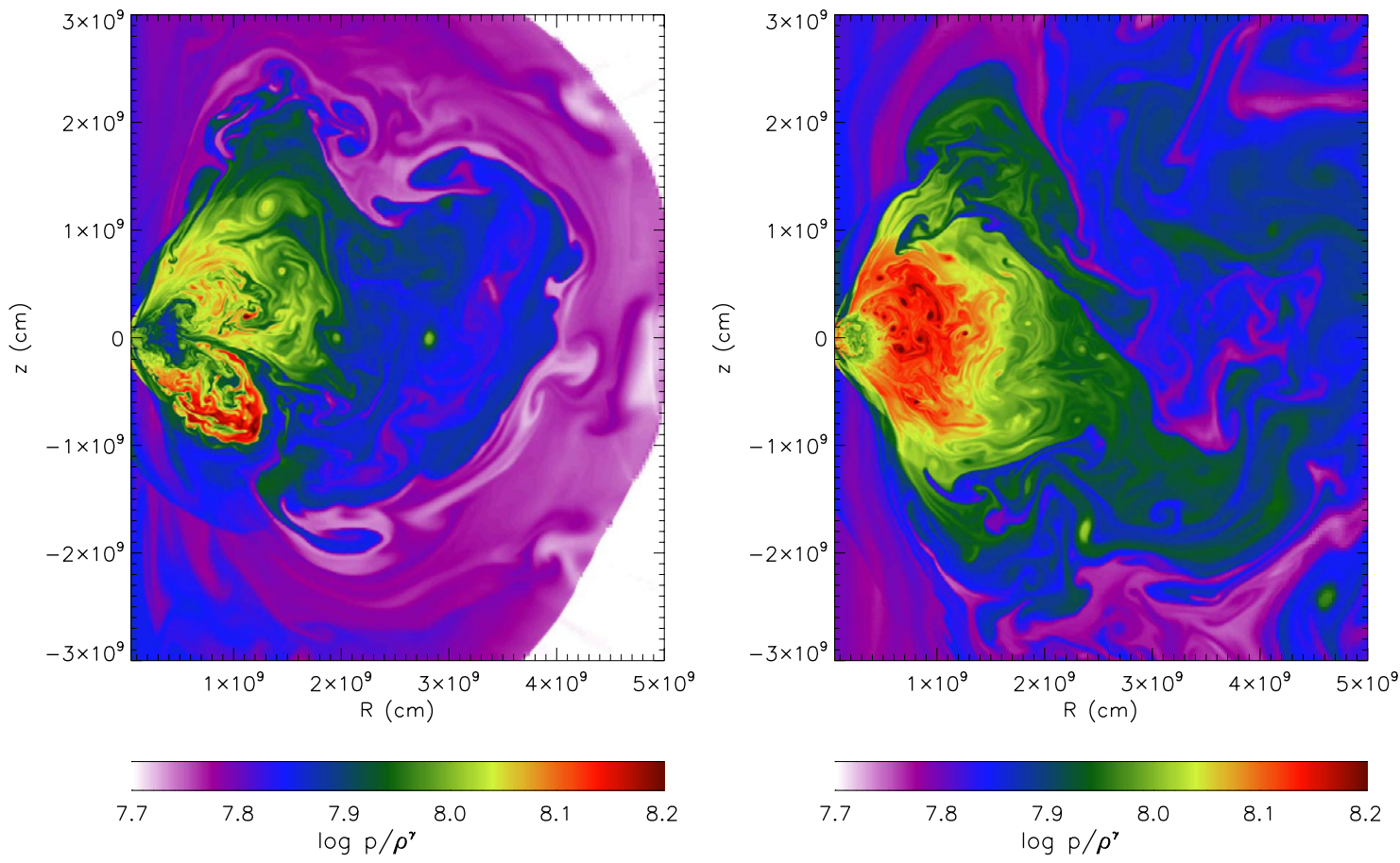

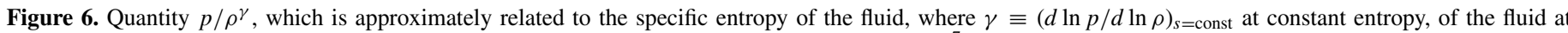

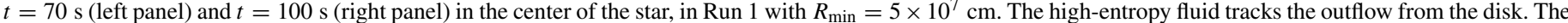

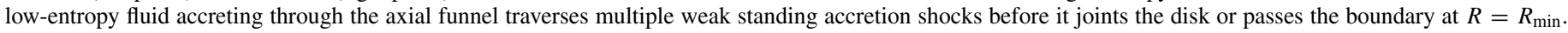
The primary, outward propagating accretion shock is visible along the right edge of the left panel.

accretion rate decline in our simulations than in those of MacFadyen et al.

The rapid temporal central accretion rate variability evident in Figure 2(c) is an outcome of hydrodynamical instabilities, such as the convective instability, near the innermost simulated radius $R_{\min }$ (see, also, MacFadyen \& Woosley 1999, who observed similar variability in Phase 0 ) and should not translate into any potential variability of the electromagnetic jet launched from radii $R \ll R_{\min }$. The nature of the variability in the inwarddirected mass flux $M$ should also be affected by the fluctuations 


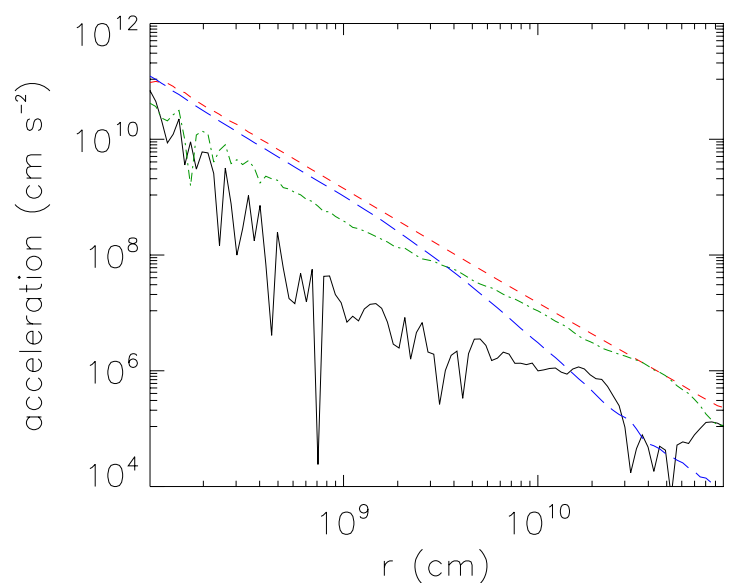

Figure 7. Gravitational field $-\partial \Phi / \partial r$ (red short-dashed line), the pressure acceleration $-\rho^{-1} \partial p / \partial r$ (green dot-dashed line), centrifugal acceleration in the radial direction $v_{\phi}^{2} \hat{R} \cdot \hat{r} / r$ (blue long-dashed line), and the sum of the gravitational, pressure, and centrifugal acceleration (black solid line) in Run 2 with $R_{\min }=10^{8} \mathrm{~cm}$. The flow is rotationally supported at $r \lesssim 3 \times 10^{9} \mathrm{~cm}$ and pressure supported at $r \gtrsim 4 \times 10^{9} \mathrm{~cm}$. We utilized the averages of $p$ and $\rho$ on spherical shells of radius $r$, and the mass-weighted averages of $\Phi$ and $v_{\phi}$ on spherical shells. Furthermore, time averaging was carried out in the interval $t=600-1000 \mathrm{~s}$

(A color version of this figure is available in the online journal.)

of the magnetic stresses (e.g., Proga \& Begelman 2003; Proga et al. 2003) and by the complex interplay of the processes associated with nuclear reactions and neutrino transport in the accretion flow (e.g., MacFadyen \& Woosley 1999; Proga et al. 2003; Nagataki et al. 2007; Lopez-Camara et al. 2009). Therefore, we caution against ascribing phenomenological significance to the accretion rate variability in Figure 2(c).

\subsection{Phase II: Funnel Outflow, Thick Disk Accretion}

The steep decline of the accretion rate seems to diminish at $\sim 200-500 \mathrm{~s}$. This behavior is suggestive of a transition into the "plateau" phase, or Phase II, of the GRB X-ray light curve, although we do not observe a robust signature of a genuine plateau in $\dot{M}(t)$ across all simulations. The simulated accretion flow appears to settle in a quasi-steady state, characterized by an axial outflow and thick equatorial disk accretion. We proceed to characterize the quasi-steady accretion flow. Figure 7, which shows the magnitudes of the various terms in the spherically averaged Euler equation and the net residual acceleration implied by the radial Euler equation, indicates that bulk of the fluid mass is rotationally supported at $r \lesssim 3 \times 10^{9} \mathrm{~cm}$ and is pressure supported at $r \gtrsim 4 \times 10^{9} \mathrm{~cm}$. The relative contribution of pressure support at radii where rotational support dominates is still substantial, $\sim 50 \%-75 \%$, consistent with the thick disk morphology with vertical scale height $h / R \sim \tan 30^{\circ}$ (Figure 5, right panel). Thus, our post-core-collapse accretion flow never resembles a thin disk. The pressure-supported atmosphere is nearly isentropic, $p \propto \rho^{\gamma}$.

In Figure 8, we show the inward directed, the outward directed, and the net mass flux flowing through spherical shells with radii $r$. After $\sim 500 \mathrm{~s}$, the net mass flux in the central $\sim 10^{9} \mathrm{~cm}$ is approximately independent of radius, which reflects a quasi-steady accretion in the inner part of the rotationally supported disk at the rate $\dot{M}_{\text {disk }} \approx 5 \times 10^{-5} M_{\odot} \mathrm{s}^{-1}$. This disk accreting in a quasi-steady state contains only $M_{\text {disk }} \sim 0.01 M_{\odot}$, which is less than $1 \%$ of the mass that remains bound to the black hole in the shock-heated, pressure-supported atmosphere atop

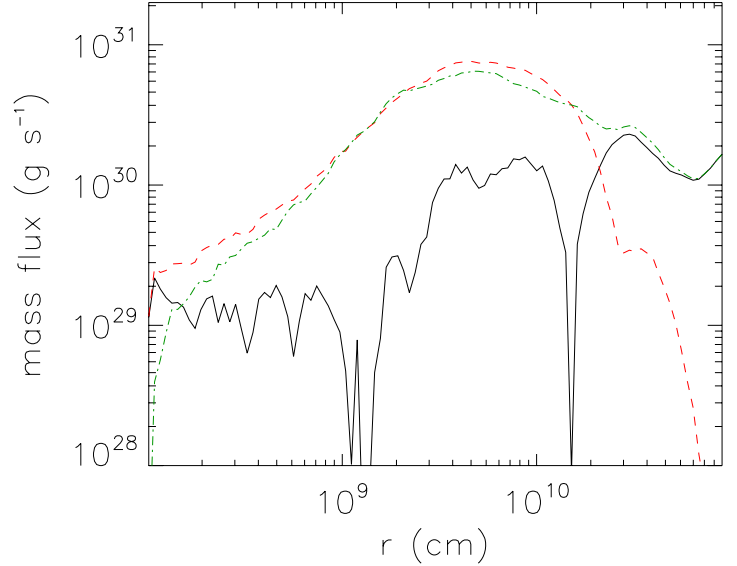

Figure 8. Rate of mass inflow (red dashed line), mass outflow (green dot-dashed line), and absolute net mass flow (black solid line) crossing a sphere of radius $r$ centered on the black hole, in Run 2 . The rates were averaged over the time interval $t=600-1000 \mathrm{~s}$. The outflow and the inflow nearly cancel over a range of radii. At the radii of the rotationally supported disk $r \lesssim 10^{9} \mathrm{~cm}$, there is net inflow at the rate $\dot{M} \sim 5 \times 10^{-5} M_{\odot} \mathrm{s}^{-1}$.

(A color version of this figure is available in the online journal.)

the rotationally supported disk. The outflow and the inflow have linearly rising accretion rates $\dot{M}_{\text {in,out }} \propto r$ and nearly cancel over the range of radii belonging to the atmosphere, just as was found in the simulated radiatively inefficient accretion flow with convection of Abramowicz et al. (2002). The structure described by a massive convective atmosphere surrounding a thick, nonradiative disk resembles the "quasistar" of Begelman et al. (2008), who envisioned the limit in which the mass of the pressure-supported envelope exceeds the mass of the black hole by a large factor.

The accretion time of the inner disk during Phase II, $t_{\text {acc }} \sim$ $M_{\text {disk }} / \dot{M}_{\text {disk }} \sim 200$ s, is shorter than duration of this phase (we end our simulation prior to the end of Phase II), hence a continuous replenishment of the inner disk must operate. The timescale on which the entire fluid mass bound to the black hole $\left(\sim 2 M_{\odot}\right)$ would accrete through the thick disk is $\sim 4 \times 10^{4} \mathrm{~s}$, though of course, not all of the mass bound at the beginning of Phase II must ultimately accrete; a large fraction could become unbound and leave in an outflow. Because of computational limitations we do not extend the simulations long enough to observe the inevitable depletion of the massive atmosphere through inner disk accretion, but in Section 4.2, we speculatively extrapolate our results into that regime.

In Figure 9, we show a large-scale $\left(\sim 10^{11} \mathrm{~cm}\right)$ view of the density and the meridional Mach number $M_{R-z} \equiv$ $\left(v_{R}^{2}+v_{z}^{2}\right)^{1 / 2} / c_{\mathrm{s}}$, where $c_{\mathrm{s}}$ is the adiabatic sound speed at $t=2000 \mathrm{~s}$ in the lowest-resolution simulation, Run 3. Meridional motions in the pressure-supported atmosphere are subsonic, confirming that large-scale infall has ceased. Because a vast fraction of the unaccreted mass is in this atmosphere, we neither observe nor anticipate the tendency of the inner disk to spread outward in the way in which an isolated thin disk would spread and how Cannizzo \& Gehrels (2009) envision. At the quasi-steady disk radii, the inner- and outward-directed mass fluxes increase outward according to $\dot{M}_{\text {in }}(r), \dot{M}_{\text {out }}(r) \propto$ $r^{1.0}-r^{1.2}$, which reflects the convective or circulatory nature of the flow. The pressure-supported atmosphere at radii $5 \times 10^{9} \mathrm{~cm} \lesssim r \lesssim 10^{10} \mathrm{~cm}$ contains about $0.5 M_{\odot}$ and exhibits a net inflow at the rate $\approx 5 \times 10^{-4} M_{\odot} \mathrm{s}^{-1}$, larger than in the inner disk; the lack of a true steady state opens the prospect 

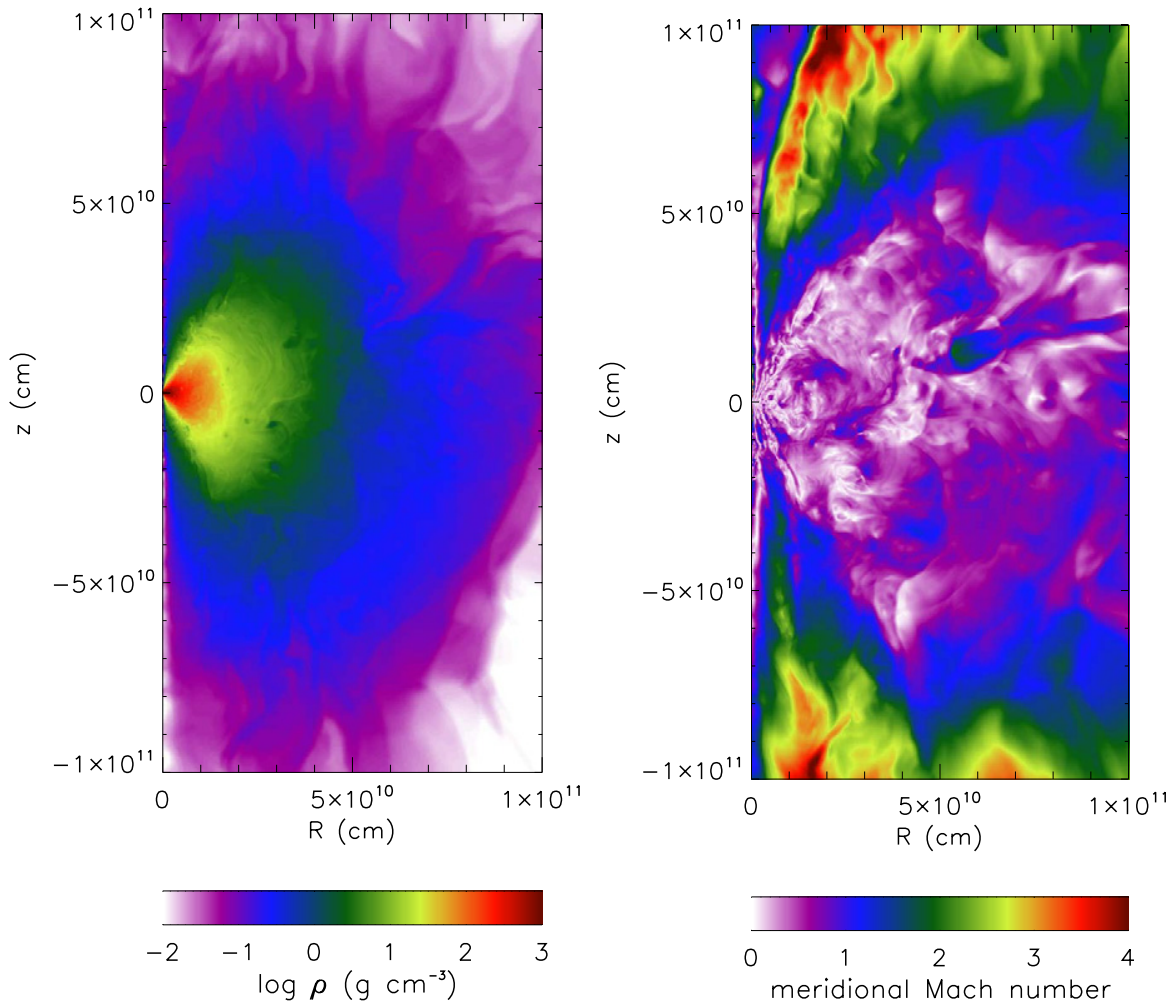

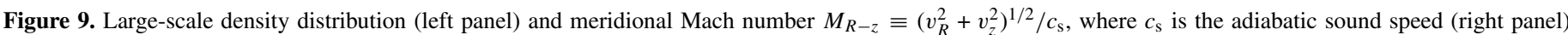

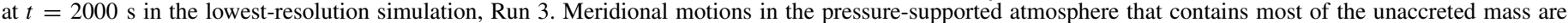
subsonic, indicating that large-scale infall has ceased. The supersonic fluid has positive Bernoulli constant and is unbound (see Figure 11).

for a late-time, high-amplitude central accretion rate variability. On the other hand, the outer atmosphere $r \gtrsim 2 \times 10^{10} \mathrm{~cm}$ containing $\sim 2 M_{\odot}$ has a net outward-directed mass flux at the rate $\dot{M}_{\text {out }} \sim(0.5-1.5) \times 10^{-3} M_{\odot} \mathrm{s}^{-1}$, though most of the outflowing mass remains gravitationally bound to the black hole.

Figure 10 shows the radial energy flow rate

$$
\begin{aligned}
\dot{E}(r)= & \int_{0}^{\pi} \rho\left[v_{r}\left(\frac{v^{2}}{2}+\frac{\gamma}{\gamma-1} \frac{P}{\rho}+\Phi\right)\right. \\
& \left.+v_{\phi} \nu \sigma \sin \theta\right] 2 \pi r^{2} \sin \theta d \theta,
\end{aligned}
$$

where $v_{r}=\left(R v_{R}+z v_{z}\right) / r, v=\left(v_{R}^{2}+v_{z}^{2}+v_{\phi}^{2}\right)^{1 / 2}, v_{\phi}=\ell / R$, and $R=r \sin \theta$. The last term in brackets is the projection onto the $r$-direction of the specific energy flux carried by the viscous torque. The entire rotationally supported region $2 \times 10^{8} \mathrm{~cm} \lesssim r \lesssim 5 \times 10^{9} \mathrm{~cm}$ exhibits a net outward-directed energy flux

$$
\dot{E}(r) \sim 10^{47} \mathrm{erg} \mathrm{s}^{-1}\left(\frac{r}{2 \times 10^{9} \mathrm{~cm}}\right)^{0.4}
$$

which in the steady state disk, $r \lesssim 10^{9} \mathrm{~cm}$, implies a mass conversion efficiency of $\dot{E} /\left(\dot{M} c^{2}\right) \sim 7 \times 10^{-4}$. In the innermost cells $r \sim 10^{8} \mathrm{~cm}$, however, there is a hint of an energy inflow.

To search for the presence of potentially unbound flows, in Figure 11, we plot the Bernoulli function, defined as the sum of the specific kinetic energy, enthalpy, and potential energy of the accretion flow (e.g., Narayan \& Yi 1994, 1995; Stone et al. 1999; Igumenshchev \& Abramowicz 1999, 2000; Blandford \&

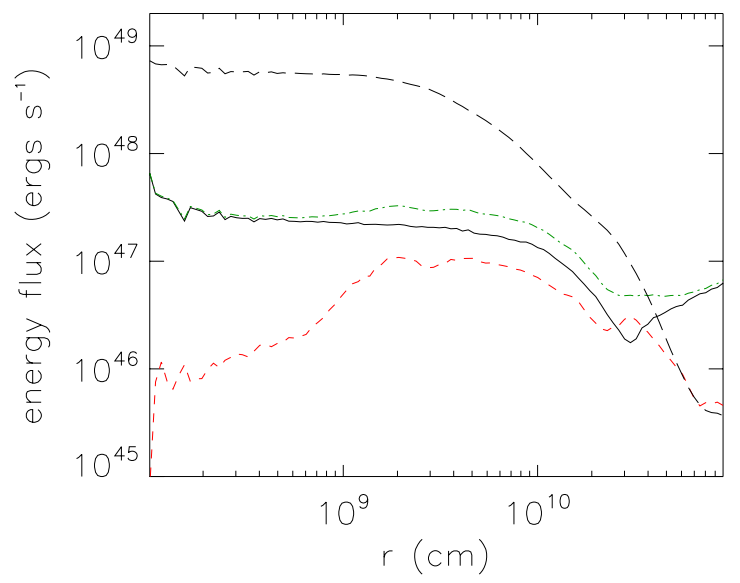

Figure 10. Rate of energy inflow (red short-dashed line), energy outflow (green dot-dashed line), and absolute net energy flow (black solid line) crossing a sphere of radius $r$ centered on the black hole in Run 2. The energy flux carried by viscosity $\rho \nu \sigma v_{\phi} \hat{R}$ was included in each case. The rates were averaged over the time interval $t=600-1000 \mathrm{~s}$. Energy outflow dominates inflow at all radii. The black long dashed line shows the product of pressure and the sound speed to which the maximum energy that can be transported by convection is proportional.

Begelman 1999, 2004) ${ }^{7}$

$$
\mathrm{Be} \equiv \frac{1}{2}\left(v_{R}^{2}+v_{z}^{2}+v_{\phi}^{2}\right)+\frac{\gamma}{(\gamma-1)} \frac{p}{\rho}+\Phi,
$$

\footnotetext{
7 Some authors also define the Bernoulli constant $b$ to equal the Bernoulli function divided by the Keplerian velocity, $b \equiv \mathrm{Be} / v_{\mathrm{K}}^{2}$ (Narayan \& Yi 1994, 1995).
} 

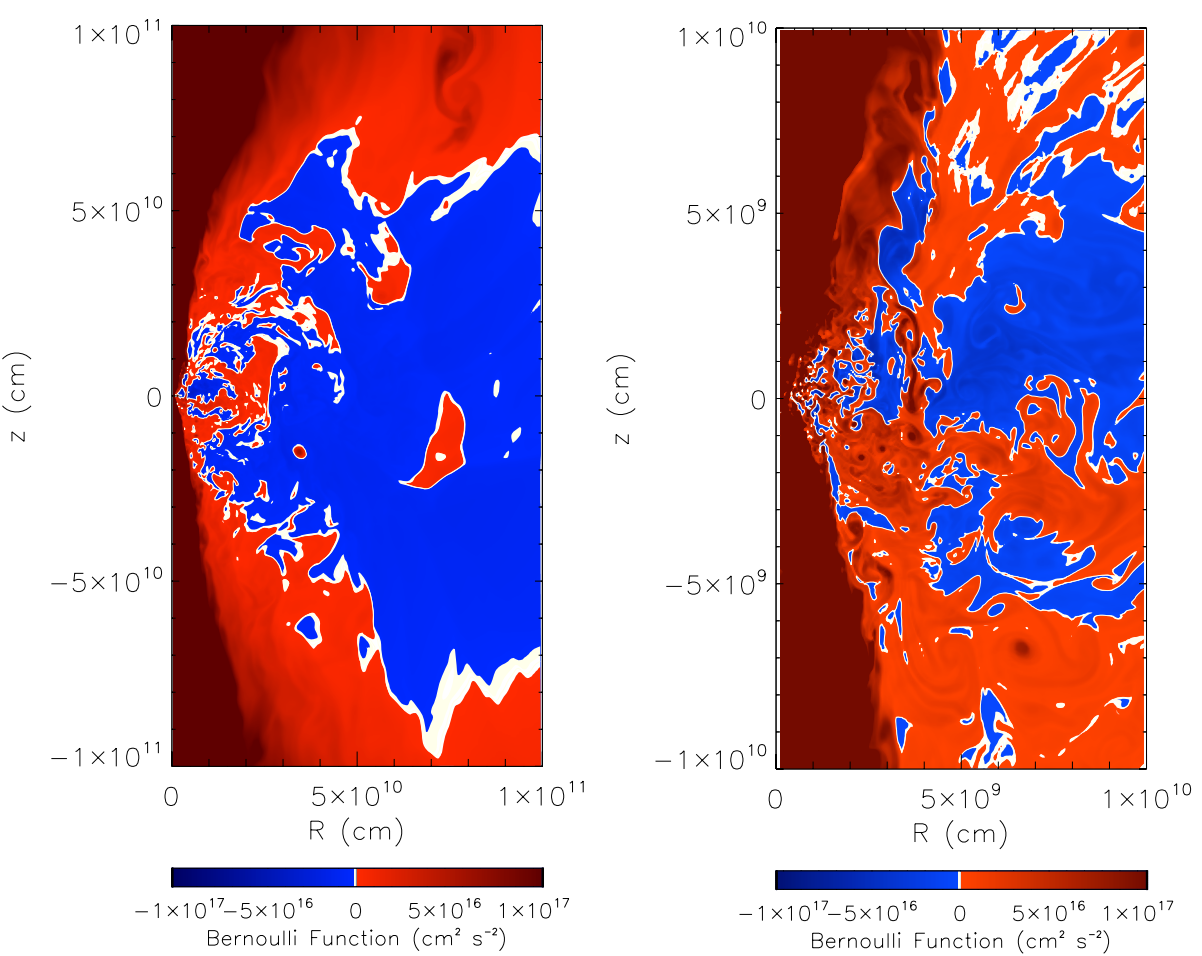

Figure 11. Value of the Bernoulli function $\mathrm{Be} \equiv E_{\mathrm{k}}+E_{\mathrm{p}}+[\gamma /(\gamma-1)] p / \rho$, where $E_{\mathrm{k}}$ and $E_{\mathrm{P}}$ denote, respectively, the specific kinetic and the potential energy, in the simulation (left panel) and in the central $10^{10} \mathrm{~cm}$ (right panel) at $t=1000 \mathrm{~s}$ in Run 2. The material with positive values of the Bernoulli function (red color) has enough energy to escape from the system.

where $\Phi$ is the total, negative gravitational potential. The structure at small radii bears only a coarse-grained resemblance to the Bernoulli function profile in the simulations of Stone et al. (1999). The entire axial funnel region, plus scattered domains within the equatorial rotationally supported and pressure-supported region, as well as the high-latitude fluid, $|z| \gtrsim 7 \times 10^{10} \mathrm{~cm}$, exhibit a positive Bernoulli function, Be $>0$, which indicates the potential for an unbound flow and an escape to infinity. The positivity of the Bernoulli function does not always necessitate escape, as such fluid elements can be buried within the massive quasi-hydrostatic envelope where they can do work on other fluid elements and mix with the $\mathrm{Be}<0$ fluid. The vertically outflowing fluid above and below the rotationally supported disk, within an angle of $\sim 20^{\circ}-25^{\circ}$ from the vertical axis, however, seems entirely unbound, in clear indication of the presence of mass loss carried by a thermally driven disk wind. Indeed, the axial outflow becomes supersonic as it propagates upward through the envelope (see Figure 9). Therefore, the system spontaneously develops an advection-dominated inflow-outflow solution (ADIOS; Blandford \& Begelman 1999, 2004). In the realistic, nonadiabatic collapsar ADIOS, the relation of the capacity to escape to the Bernoulli function is modified by the cooling due to neutrino emission and the endothermic nuclear disintegration, which could subvert escape, and by the heating due to the exothermic nuclear recombination in the outflow (Pruet et al. 2003; Surman et al. 2006; Fujimoto et al. 2007; Nagataki et al. 2007; Maeda \& Tominaga 2009), which could promote escape.

\section{DISCUSSION}

The primary limitations of the numerical model presented here include: (1) the lack of simulation coverage of the hot inner disk, $r \lesssim 5 \times 10^{7} \mathrm{~cm}$, where neutrino and nuclear physics influence the thermodynamics of the flow, (2) the limited adequacy of the Navier-Stokes viscous fluid dynamics to approximate the dynamics of a realistic magnetized, radiationdominated fluid, (3) the lack of modeling of the axial relativistic jet and its enveloping cocoon, and (4) the lack of coverage of the very late evolution, $t \gtrsim 10^{4} \mathrm{~s}$, when the GRB X-ray light curve exhibits single or multiple breaks with the tendency toward a steepening of the luminosity decline. We defer an exploration of the limitations (2) and (3) for future work, and here briefly and speculatively address limitations (1) and (4). In Section 4.1, we crudely take into account the energy loss to neutrino emission in the accretion-shock-heated flow and estimate the time at which the central accretion rate commences to decline steeply (the transition from Phase 0 to Phase I). In Section 4.2, we discuss the implications of mass loss for the long-term evolution of accretion rate.

\subsection{Triggering of the Steep Decline in GRBs}

The composite $\gamma$-ray and X-ray GRB light curve starts declining abruptly and steeply after an initial period of steady luminosity that lasts for tens of seconds. Here, we attempt to shed light on the transition from the quasi-steady activity of the central engine to the steeply declining regime. Continuing to work within the paradigm in which the luminosity is proportional to the central accretion rate, we suggest that the steep decline is triggered by a rapid outward expansion of an accretion shock through the infalling material that feeds a convective, rotationally supported thick accretion disk. This is consistent with the conclusion of Barniol Duran \& Kumar (2009), who ruled out mechanisms for powering the rapid decline that are intrinsic to a single emitting and cooling element, and by elimination inferred that the central engine (e.g., an accreting black hole) remains active during the steeply declining phase (but see Genet \& Granot 2008, who showed that the high-latitude emission from a sequence of such elements, or "pulses," could fit the decline). 
Because in our adiabatic simulations we do not resolve the innermost radii $r \lesssim 5 \times 10^{7} \mathrm{~cm}$ and do not simulate the nuclear disintegration, neutrino emission, neutrino capture on nucleons, and neutrino annihilation, that occur in the hot $\left(T \gtrsim 10^{10} \mathrm{~K}\right)$ plasma at these radii, some of the forthcoming conclusions will be obtained with the aid of a simple analytical model for the thermodynamic evolution of the inner neutrino-cooling region (see also Beloborodov 2008).

We have seen that in the adiabatic simulations, the accretion shock appears when the infalling material has enough angular momentum to be rotationally supported at the innermost resolved radius. From there on, the accretion shock expands outward rapidly and sweeps through the star. The shocked fluid is additionally heated as a result of the viscous dissipation in the thick disk. The energy produced during the accretion of the thick disk is advected or convected radially outward by the disk "wind" (Figure 6) which distributes it throughout the mostly pressure-supported torus of shocked fluid. In the adiabatic regime, after the gravitational potential becomes dominated by the black hole while the mass infall rate declines rapidly, it seems that an outward expansion of the hot torus bounded by the accretion shock is inevitable immediately following shock formation. In the nonadiabatic regime, the expansion of the shock may be delayed by losses associated with nuclear disintegration and neutrino emission. If indeed, in general, a sudden and rapid drop in the central accretion rate accompanies the shock expansion, then to estimate the onset of the steep decline, one must identify the instance at which the losses become inefficient and the accretion shock, aided by the viscous energy injection, can start traveling outward.

Consider a fluid element with specific angular momentum $\ell=10^{17} \ell_{17} \mathrm{~cm}^{2} \mathrm{~s}^{-1}$; its circularization radius around the black hole of mass $M_{\mathrm{BH}}=10 M_{1} M_{\odot}$ is

$$
r_{\text {circ }} \sim \frac{\ell^{2}}{G M_{\mathrm{BH}}} .
$$

If the material with density $\rho=10^{8} \rho_{8} \mathrm{~g} \mathrm{~cm}^{-3}$ arrives at the shock from a free fall from infinity and the gravitational energy density $G M_{\mathrm{BH}} \rho / r_{\text {circ }}$ is converted into the energy density in radiation $a T^{4}$, where $a$ is the radiation constant, the post-shock temperature is given by

$$
\begin{aligned}
T_{\text {shock }} & \sim\left(\frac{G M_{\mathrm{BH}}}{\ell}\right)^{1 / 2}\left(\frac{7 \rho}{a}\right)^{1 / 4} \\
& \sim 6.4 \times 10^{10} \mathrm{~K} \frac{M_{1}^{1 / 2} \rho_{8}^{1 / 4}}{\ell_{17}^{1 / 2}},
\end{aligned}
$$

where we have taken the density jump across the shock to be $\sim 7$, appropriate if the fluid velocity is Newtonian and the postshock fluid is radiation-dominated. The latter in particular is a crude approximation; Chen \& Beloborodov (2007) show that the pressure due to baryons and electrons and positrons can be comparable to and larger than the radiation pressure in the disk midplane.

Nagataki et al. (2007) find that almost all of the energy emitted by neutrinos in the hot, rotationally supported torus, comes from pair capture on free nucleons (the Urca process), ${ }^{8}$ for which the

\footnotetext{
8 In the calculation of Lopez-Camara et al. (2009), the cooling due to neutrino emission from pair annihilation dominates at early times $t=0.2 \mathrm{~s}$ and radii $R \lesssim 2 \times 10^{7} \mathrm{~cm}$ on the equatorial plane; at later times, the cooling from pair annihilation is comparable to the cooling from pair capture.
}

approximate cooling rate is (Qian \& Woosley 1996; Popham et al. 1999, and references therein)

$$
q_{e N}=9 \times 10^{25} \rho_{8} T_{10}^{6} X_{\text {nuc }} \operatorname{erg~cm}^{-3} \mathrm{~s}^{-1},
$$

where $T=10^{10} T_{10} \mathrm{~K}$ is the plasma temperature, and $X_{\text {nuc }}$ is the free nucleon fraction in nuclear statistical equilibrium (NSE), which is approximated via

$$
X_{\text {nuc }} \approx \min \left[1,8.7 \rho_{8}^{-3 / 4} T_{10}^{9 / 8} \exp \left(-6.1 / T_{10}\right)\right] .
$$

As long as the cooling time

$$
t_{\mathrm{cool}}=\frac{a T^{4}}{q},
$$

where $q$ is the net energy loss rate, which includes the pair capture term $q_{e N}$ and other contributions, is shorter than the age of the collapse $t$, the accretion shock is confined near the black hole, the flow crossing the shock is highly supersonic, and rapid accretion onto the central object is possible.

When the cooling time exceeds the age of the collapse, $t_{\text {cool }}>t$, the accretion shock expands outward. It seems that in the specific case of the collapse of a 16TI-model star, the pair capture neutrino emission indeed dominates losses until the cooling is no longer able to prevent outward expansion of the shock. When the post-shock temperature drops below $\sim 9 \times 10^{9} \mathrm{~K}$, which happens due to the decrease in the free nucleon abundance under NSE conditions that are still satisfied until the temperature drops to $T_{\text {min,NSE }} \sim 4 \times 10^{9} \mathrm{~K}$, the losses from nuclear disintegration and from neutrino emission from pair annihilation exceed those from pair capture, but they are not able to prevent shock expansion.

Consider an initial stellar density profile of the form $\rho \propto r^{-\tau}$ in which the gravity is dominated by the mass closer to the center, and let

$$
M(r)=M_{0}\left(\frac{r}{r_{0}}\right)^{3-\tau}
$$

denote the pre-collapse mass profile, where $M_{0}$ and $r_{0}$ denote the stellar mass and radius, respectively (we ignore the departure of the density profile from a single power law near the stellar surface). The free-fall time from radius $r$ is given by $t_{\mathrm{ff}}(r) \sim$ $\left[r^{3} / G M(r)\right]^{1 / 2}$. This relation can be inverted to obtain the radius, defined via $t_{\mathrm{ff}}\left(r_{\mathrm{ff}}\right)=t$, from which the freely falling material is reaching the center at time $t$,

$$
r_{\mathrm{ff}}(t)=\left(G M_{0} t^{2} r_{0}^{\tau-3}\right)^{1 / \tau}
$$

The mass of the black hole grows in time and approximately equals

$$
\begin{aligned}
M_{\mathrm{BH}}(t) & \sim M\left[r_{\mathrm{ff}}(t)\right] \\
& \sim G^{3 / \tau-1} M_{0}^{3 / \tau} r_{0}^{3(1-3 / \tau)} t^{2(3 / \tau-1)} .
\end{aligned}
$$

Assuming that $r_{\text {circ }} \ll r_{\text {ff }}$, the pre-shock density of infalling fluid at $r_{\text {circ }}$ at time $t$ since the beginning of the explosion approximately equals the mass infall rate $\sim M\left[r_{\mathrm{ff}}(t)\right] / t$ divided by the shock area $\sim 4 \pi r_{\text {circ }}^{2}$ and multiplied by the infall velocity $\sim\left(G M_{\mathrm{BH}} / r_{\text {circ }}\right)^{1 / 2}$,

$$
\begin{aligned}
\rho(t) & \sim \frac{M\left[r_{\mathrm{ff}}(t)\right]}{4 \pi\left[G M_{\mathrm{BH}}(t) r_{\mathrm{circ}}^{3}\right]^{1 / 2} t} \\
& \sim \frac{G^{1-6 / \tau} M_{0}^{6 / \tau} r_{0}^{6(1-3 / \tau)} t^{12 / \tau-5}}{4 \pi \ell(t)^{3}} .
\end{aligned}
$$


We further assume that

$$
\ell(r) \sim \ell_{0}\left[\frac{M(r)}{M_{0}}\right]^{\eta} \mathrm{cm}^{2} \mathrm{~s}^{-1},
$$

implying that

$$
\ell(t) \sim \ell_{0}\left(G M_{0}\right)^{(3 / \tau-1) \eta} r_{0}^{3(1-3 / \tau) \eta} t^{2(3 / \tau-1) \eta} .
$$

Kumar et al. (2008b) find that $\tau \approx 2.5$ throughout the bulk of the star for the model 16TI of Woosley \& Heger (2006) that we utilize and we adopt this value. We further find that $\eta \approx 2.5$ is consistent with the rotational profile of the model $16 \mathrm{TI}$ in the range $4 M_{\odot} \lesssim M(r) \lesssim 10 M_{\odot}$. We set $r_{0}=10^{10} \mathrm{~cm}$, and $M_{0}=10 M_{\odot}$ and $\ell_{0}=10^{17.8} \mathrm{~cm}^{2} \mathrm{~s}^{-1}$, which approximate the mass and angular profile of the model 16TI, and substitute $r=r(t)$ in Equation (29) and substitute $\ell[r(t)]$ in Equation (28) to obtain

$$
\begin{aligned}
\rho & \sim \frac{M_{0}^{9 / 10} r_{0}^{33 / 10}}{4 \pi G^{1 / 10} \ell_{0}^{3} t^{16 / 5}} \\
& \sim 10^{10} t^{-16 / 5} \mathrm{~g} \mathrm{~cm}^{-3},
\end{aligned}
$$

where in the last expression, $t$ is given in seconds.

Combining Equations (21)-(24) and (31), the ratio of the cooling time for pair capture only, $q=q_{e N}$, to the age of the collapse reads

$$
\frac{t_{\mathrm{cool}}}{t} \sim \frac{2 \times 10^{-6} t^{22 / 5}}{\min \left[1,17.5 t^{93 / 80} \exp \left(-0.27 t^{11 / 10}\right)\right]} .
$$

The ratio rises rapidly in time and becomes unity $t_{\text {cool }} / t \sim 1$ at

$$
t_{\mathrm{decl}} \approx 20 \mathrm{~s} .
$$

At this point, the material circularizing at $r_{\text {circ }}$ is no longer able to cool by neutrino emission. Therefore, we expect that in a realistic star corresponding to the model 16TI, the accretion rate starts to decline steeply at $t_{\mathrm{decl}} \sim 20 \mathrm{~s}$ after the explosion, when the mass of the black hole is $\sim 9 M_{\odot}$ and post-shock temperature is $\lesssim 10^{10} \mathrm{~K}$. Since the initial mass of the black hole, if taken to equal the mass of the iron core, is $M_{\mathrm{BH} \text {,init }} \sim 1.5 M_{\odot}$, the implied average accretion rate preceding the decline, $\langle\dot{M}\rangle=$ $\left[M_{\mathrm{BH}}\left(t_{\mathrm{decl}}\right)-M_{\mathrm{BH} \text {,init }}\right] / t_{\mathrm{decl}} \sim 0.4 M_{\odot} \mathrm{s}^{-1}$, is larger than the accretion rate $\sim(0.1-0.2) M_{\odot} \mathrm{s}^{-1}$ observed in our simulations and those of MacFadyen \& Woosley (1999) and Nagataki et al. (2007), though it is consistent with the accretion rate in the first $0.3 \mathrm{~s}$ in the simulation of Proga et al. (2003) and in the first $0.4 \mathrm{~s}$ in the simulation of Lopez-Camara et al. (2009). Our analytical model possibly overestimates the infall rate as it does not take into account the initial hydrostatic pressure gradients that delay the collapse in the simulations and in real GRB progenitors.

If this model for the triggering of the steep decline of the central accretion rate is correct, and if the onset of the steep decline of the accretion rate implies a termination of the observable prompt $\gamma$-ray emission, then the duration of the prompt emission in long GRBs produced by black hole-forming core-collapse events should be anti-correlated with the angular momentum of the progenitor envelope. High-angular-momentum envelopes circularize at large radii where low virial temperatures imply post-shock adiabaticity and an earlier heating of the infalling envelope by the outward expanding accretion shock and disk outflows. Low-angular-momentum envelopes may circularize at radii where the high virial temperatures imply rapid cooling over several tens of seconds after the initial collapse. The cooling allows the accretion shock to remain confined longer at radii where the free-fall velocity of the infalling envelope is highly supersonic and a high central accretion rate is possible, barring, of course, another process, such the electromagnetically driven outflow observed in, e.g., Proga et al. (2003), Nagataki et al. (2007), and Komissarov \& Barkov (2009), that could suppress central accretion.

\subsection{The Long-term Evolution}

The period of quasi-steady or gradually declining luminosity in GRB X-ray light curves lasts for $\sim 10^{3}-10^{4} \mathrm{~s}$ (Phase II). At the end of this period, a steeper decline resumes (Phase III), but with the shallower slope $L_{\mathrm{X}} \propto t^{-1.2}$ than in the steeply declining regime of Phase I. Occasionally, at $t \sim 10^{4}-10^{5} \mathrm{~s}$, an even steeper decline $L_{\mathrm{X}} \propto t^{-2}$ takes over (Phase IV in the nomenclature of Zhang et al. 2006). If the steepening of the light curve reflects an underlying decline of the central accretion rate, what process is responsible for this decline? Possibilities include a transformation of the character of the accretion flow due to an internal redistribution of material inside the accreting envelope and a depletion of the mass reservoir that feeds the central accretion.

At the densities of the accreting, pressure-supported envelope, which are $>10^{-3} \mathrm{~g} \mathrm{~cm}^{-3}$ at the outer boundary of the simulation box at the end of each run, the radiation is effectively trapped and internal radiation transfer in the disk and the envelope is not important on the timescales on which X-ray light curve data are available. It also seems that given the near-hydrodynamic equilibrium state at $t \sim 10^{3} \mathrm{~s}$, any longer-term internal hydrodynamic redistribution of material between the disk and the envelope should be very gradual, so such a hydrodynamic redistribution is probably not a candidate for the steepening that marks the transition from Phase II to Phase III or that which marks the transition from Phase III to Phase IV. Depletion of the reservoir consisting of the rotationally supported disk and the pressure-supported atmosphere could occur due to the accretion of the fluid into the black hole ("draining"), due to a hydrodynamic outflow launched from the surface of the thick disk and escaping through the axial funnel region ("venting"), and due to a radiatively driven mass loss in the photosphere of the envelope ("blowoff").

In the absence of mass loss to unbound flows, the timescale on which the gravitationally bound envelope drains into the black hole, estimated from the bound envelope mass $\left(M_{\text {env }} \sim 2 M_{\odot}\right)$ and the accretion rate $\left(\dot{M} \sim 5 \times 10^{-5} M_{\odot} \mathrm{s}^{-1}\right.$; see Section 3.3) at $t=600-1000 \mathrm{~s}$ in Run 2 is

$$
t_{\mathrm{acc}} \sim \frac{M_{\mathrm{env}}}{\dot{M}} \sim 4 \times 10^{4} \mathrm{~s} .
$$

This timescale is somewhat longer but within uncertainties consistent with the timescale of the initial steepening of the light curve at the transition from Phase II to Phase III. If the evolution of the envelope under draining is self-similar $\dot{M} \propto M_{\text {env }}$, one might expect an exponential decline of the accretion rate; such a self-similarity, however, is not necessarily expected.

The positive Bernoulli function of the fluid in the region of the axial funnel in Figure 11 and the mass influx and outflux that increases with radius in Figure 8 suggest the possibility that the dominant depletion may not be to accretion into the black hole, but instead to the loss exacted by the wind launched 
thermally from the surface of the thick, convective, rotationally supported disk. The peak net outflow rate at $r \sim 3 \times 10^{10} \mathrm{~cm}$ in Run 2 is $\dot{M}_{\text {out }} \sim 10^{-3} M_{\odot} \mathrm{s}^{-1}$, which implies a short depletion timescale of $t_{\text {loss }} \sim 500 \mathrm{~s}$. This timescale, however, is almost certainly an overestimate given that the high outflow rate may be a transient associated with the incomplete readjustment to the passage and breakout of the primary accretion shock. It seems evident, however, that the draining into the black hole and the mass loss to hydrodynamically and thermally driven outflows from the surface of the thick disk and the massive envelope can provide explanations of the termination of quasi-steady accretion marking the Phase II to Phase III transition.

Convective energy transport in the massive, pressuresupported envelope can continue out to some critical radius where the convective motions become supersonic, resulting in shocks, or where radiation diffusion across the convective cells thwarts the convective instability. Outside of this radius, energy is transported either radiatively or by non-convective bulk motions. Since the energy flux is a factor of $10^{7}-10^{8}$ above the Eddington limit, radiation pressure accelerates the fluid outward, resulting in a supersonic wind (see, e.g., Shaviv 2001; Owocki et al. 2004; van Marle et al. 2008). The wind mass-loss rate is limited by energy conservation $\frac{1}{2} \dot{M}_{\text {wind }} v_{\text {esc }}^{2} \leqslant L$, and if the wind driving is radiative, momentum conservation, $\dot{M}_{\text {wind }} v_{\infty} \leqslant L / c$, where $v_{\text {esc }}$ is the escape velocity from the critical radius, and $v_{\infty}$ is the velocity of the wind at infinity. To our best knowledge, the mechanics of mass loss in this extremely super-Eddington regime have not been explored. There is the possibility that the atmospheric mass loss occurs on a timescale compatible with the final steepening of the GRB X-ray light curve, at the Phase III to Phase IV transition. Alternatively, as we have argued above, the Phase II to Phase III transition, and the Phase III to Phase IV transition, could both be caused by nonradiative losses (the draining into the black hole and venting in the axial funnel), but longer-term simulations are required to check this possibility.

\section{CONCLUSIONS}

We have conducted hydrodynamic simulations of the viscous post-core-collapse accretion of a rapidly rotating $\sim 14 M_{\odot}$ Wolf-Rayet star of Woosley \& Heger (2006) onto the central black hole. The axially symmetric simulations were carried out for up to $2000 \mathrm{~s}$ and resolved the radii down to $5 \times 10^{7} \mathrm{~cm}$ where the collapsing stellar material circularizes around the black hole. The evolution of the central accretion rate in the simulations resembles the evolution of the observed GRB $\mathrm{X}$-ray luminosity, which lends support to the hypothesis (Kumar et al. 2008a, 2008b) that the X-ray luminosity is proportional to the rate with which stellar material accretes onto the black hole. We have identified three phases in the evolution of the accretion rate in our simulations, which appear to correspond to Phases 0 (the prompt phase), Phase I, and Phase II in the nomenclature of Zhang et al. (2006).

In the initial phase that in the simulations lasts $37-52 \mathrm{~s}$, the accretion of low-angular-momentum material is quasiradial for $r>5 \times 10^{7} \mathrm{~cm}$ and occurs at quasi-constant rate of $\sim 0.2 M_{\odot} \mathrm{s}^{-1}$. The end of this phase is marked by the formation of an accretion shock at the smallest resolved radii. The shock immediately propagates radially outward through the supersonically infalling stellar envelope. Simultaneously with the formation and the outer movement of the accretion shock, the accretion rate drops suddenly and precipitously. We argue that the somewhat late onset of the accretion shock is an artifact of our not resolving the innermost two decades in radius outside the black hole's gravitational radius.

We supplement the simulations with an analytical model of the innermost accretion disk not resolved in the simulations, and suggest that the accretion shock forms early, within a fraction of the first second of the formation of the black hole, as several published simulations of the innermost neutrino-cooled region have shown, but only starts to propagate outward after $20 \mathrm{~s}$, when the material that is reaching the equatorial plane has enough angular momentum to circularize at radii where the virial temperature is below $\sim 10^{10} \mathrm{~K}$ and the cooling by neutrino emission is suppressed.

During the second phase characterized by a steep decline $\propto t^{-2.7}$ of the accretion rate that lasts $\sim 500 \mathrm{~s}$, the accretion shock sweeps through the star, but a supersonic accretion of the shocked fluid in the axial funnel region proceeds unabated, at least in our simulations where the funnel has not been heated to high temperatures by the relativistic jet. The thick disk containing rotationally supported and pressure-supported fluid is convective; a high-entropy outflow from the inner, rotationally supported region follows the accretion shock on its traversal through the star but remains bound within the star and appears to form a large-scale circulation pattern. The steepness of the accretion rate decline seems to be the consequence of a rapid hydrodynamic readjustment of the shocked, convective, and circulating stellar envelope.

The steep decline of the accretion rate slows down or stalls after $\sim 600 \mathrm{~s}$, which appears to reflect the settling of a fraction of the stellar envelope in the state of near-hydrostatic equilibrium. The inner, rotationally supported thick disk contains $\sim 1 \%$ of the mass of the unaccreted envelope and extends to $\sim 3 \times 10^{9} \mathrm{~cm}$. The thick disk is surrounded by a much more massive, pressuresupported atmosphere, which acts as a mass supply to the thick disk. At no point do we find evidence for the extended thin disk envisioned by Cannizzo \& Gehrels (2009). The fluid above and below the thick disk is mostly unbound and the simulations thus exhibit a form of a "disk wind."

We speculate that depletion of the envelope through accretion onto the black hole or mass loss in thermal outflows or winds could be responsible for the renewed steepening of the GRB $\mathrm{X}$-ray light curve after $10^{3}-10^{4} \mathrm{~s}$. More speculatively, the additional steepening of the light curve occasionally observed after $10^{4}-10^{5} \mathrm{~s}$ could be due to a pervasive thermal or radiatively driven mass loss in the outer layers of the atmosphere.

We thank Ramesh Narayan and Craig Wheeler for encouraging this research at an early stage. We also thank Stan Woosley and Alex Heger for making their pre-supernova stellar model 16TI available. In addition, we thank Rodolfo Barniol Duran and Rongfeng Shen for valuable discussions. The software used in this work was in part developed by the DOE-supported ASC/ Alliance Center for Astrophysical Thermonuclear Flashes at the University of Chicago. The authors acknowledge the Texas Advanced Computing Center (TACC) at the University of Texas at Austin for providing high-performance computing resources that have contributed to this research. This material is based upon work supported under a National Science Foundation Graduate Research Fellowship awarded to C.C.L. M.M. acknowledges support from NSF grant AST-0708795 and P.K. acknowledges support from NSF grant AST-0909110. 


\section{REFERENCES}

Abramowicz, M. A., Igumenshchev, I. V., Quataert, E., \& Narayan, R. 2002, ApJ, 565,1101

Balbus, S. A., \& Hawley, J. F. 1998, Rev. Modern Phys., 70, 1

Balbus, S. A., \& Hawley, J. F. 2002, ApJ, 573, 749

Barkov, M. V., \& Komissarov, S. S. 2008, in AIP Conf. Proc. 1085, High Energy Gamma-Ray Astronomy: Proc. 4th Int. Meeting on High Energy GammaRay Astronomy, ed. F. A. Aharonian, W. Hofmann, \& F. Rieger (Melville, NY: AIP), 608

Barniol Duran, R., \& Kumar, P. 2009, MNRAS, 395, 955

Begelman, M. C., Rossi, E. M., \& Armitage, P. J. 2008, MNRAS, 387, 1649

Beloborodov, A. M. 2008, in AIP Conf. Proc. 1054, Cool Discs, Hot Flows: The Varying Faces of Accreting Compact Objects, ed. M. Axelsson (Melville, NY: AIP), 51

Blandford, R. D., \& Begelman, M. C. 1999, MNRAS, 303, L1

Blandford, R. D., \& Begelman, M. C. 2004, MNRAS, 349, 68

Bucciantini, N., Quataert, E., Arons, J., Metzger, B. D., \& Thompson, T. A 2007, MNRAS, 380, 1541

Bucciantini, N., Quataert, E., Metzger, B. D., Thompson, T. A., Arons, J., \& Del Zanna, L. 2009, MNRAS, 396, 2038

Burrows, D. N., et al. 2005, Science, 309, 1833

Cannizzo, J. K., \& Gehrels, N. 2009, ApJ, 700, 1047

Christodoulou, D. M., Contopoulos, J., \& Kazanas, D. 2003, ApJ, 586, 372

Chen, W.-X., \& Beloborodov, A. M. 2007, ApJ, 657, 383

Di Matteo, T., Perna, R., \& Narayan, R. 2002, ApJ, 579, 706

Duncan, R. C., \& Thompson, C. 1992, ApJ, 392, L9

Falcone, A. D., et al. 2006, ApJ, 641, 1010

Fryxell, B., et al. 2000, ApJS, 131, 273

Fujimoto, S.-i., Hashimoto, M.-a., Kotake, K., \& Yamada, S. 2007, ApJ, 656, 382

Genet, F., \& Granot, J. 2008, arXiv:0812.4677

Hirose, S., Krolik, J. H., \& Blaes, O. 2009, ApJ, 691, 16

Igumenshchev, I. V. 2002, ApJ, 577, L31

Igumenshchev, I. V., \& Abramowicz, M. A. 1999, MNRAS, 303, 309

Igumenshchev, I. V., \& Abramowicz, M. A. 2000, ApJS, 130, 463

Igumenshchev, I. V., Abramowicz, M. A., \& Narayan, R. 2000, ApJ, 537, L27

Igumenshchev, I. V., Narayan, R., \& Abramowicz, M. A. 2003, ApJ, 592, 1042

Janiuk, A., \& Proga, D. 2008, ApJ, 675, 519

Kohri, K., Narayan, R., \& Piran, T. 2005, ApJ, 629, 341

Komissarov, S. S., \& Barkov, M. V. 2007, MNRAS, 382, 1029

Komissarov, S. S., \& Barkov, M. V. 2009, MNRAS, 397, 1153

Kumar, P., Narayan, R., \& Johnson, J. L. 2008a, Science, 321, 376

Kumar, P., Narayan, R., \& Johnson, J. L. 2008b, MNRAS, 388, 1729

Landau, L. D., \& Lifshitz, E. M. 1959, Fluid Mechanics (Oxford: Pergamon Press)

Lazzati, D., \& Perna, R. 2007, MNRAS, 375, L46

Lazzati, D., Perna, R., \& Begelman, M. C. 2008, MNRAS, 388, L15

Lee, W. H., \& Ramirez-Ruiz, E. 2006, ApJ, 641, 961

Lopez-Camara, D., Lee, W. H., \& Ramirez-Ruiz, E. 2009, ApJ, 692, 804

MacFadyen, A. I. 2003a, in AIP Conf. Proc. 662, Gamma-Ray Burst and Afterglow Astronomy 2001: A Workshop Celebrating the First Year of the HETE Mission, ed. G. R. Ricker \& R. K. Vanderspek (Melville, NY: AIP), 202
MacFadyen, A. I. 2003b, in ESO Astrophys. Symp., From Twilight to Highlight: The Physics of Supernovae: Proc. ESO/MPA/MPE Workshop, ed. W. Hillenbrandt \& B. Leibundgut (Berlin: Springer), 97

MacFadyen, A. I., \& Woosley, S. E. 1999, ApJ, 524, 262

MacFadyen, A. I., Woosley, S. E., \& Heger, A. 2001, ApJ, 550, 410

Maeda, K., \& Tominaga, N. 2009, MNRAS, 394, 1317

Morsony, B. J., Lazzati, D., \& Begelman, M. C. 2007, ApJ, 665, 569

Nagataki, S., Takahashi, R., Mizuta, A., \& Takiwaki, T. 2007, ApJ, 659, 512

Narayan, R., Igumenshchev, I. V., \& Abramowicz, M. A. 2000, ApJ, 539, 798

Narayan, R., Piran, T., \& Kumar, P. 2001, ApJ, 557, 949

Narayan, R., \& Yi, I. 1994, ApJ, 428, L13

Narayan, R., \& Yi, I. 1995, ApJ, 444, 231

Nousek, J. A., et al. 2006, ApJ, 642, 389

O’Brien, P. T., et al. 2006, ApJ, 647, 1213

Ohsuga, K., Mori, M., Nakamoto, T., \& Mineshige, S. 2005, ApJ, 628, 368

Owocki, S. P., Gayley, K. G., \& Shaviv, N. J. 2004, ApJ, 616, 525

Perna, R., Armitage, P. J., \& Zhang, B. 2006, ApJ, 636, L29

Piro, A. L., \& Pfahl, E. 2007, ApJ, 658, 1173

Popham, R., Woosley, S. E., \& Fryer, C. 1999, ApJ, 518, 356

Pringle, J. E. 1981, ARA\&A, 19, 137

Proga, D., \& Begelman, M. C. 2003, ApJ, 592, 767

Proga, D., MacFadyen, A. I., Armitage, P. J., \& Begelman, M. C. 2003, ApJ, 599, L5

Pruet, J., Woosley, S. E., \& Hoffman, R. D. 2003, ApJ, 586, 1254

Qian, Y.-Z., \& Woosley, S. E. 1996, ApJ, 471, 331

Ramirez-Ruiz, E., Celotti, A., \& Rees, M. J. 2002, MNRAS, 337, 1349

Rockefeller, G., Fryer, C. L., \& Li, H. 2006, arXiv:astro-ph/0608028

Ryu, D., \& Goodman, J. 1992, ApJ, 388, 438

Shakura, N. I., \& Sunyaev, R. A. 1973, A\&A, 24, 337

Shaviv, N. J. 2001, MNRAS, 326, 126

Stone, J. M., \& Balbus, S. A. 1996, ApJ, 464, 364

Stone, J. M., Pringle, J. E., \& Begelman, M. C. 1999, MNRAS, 310, 1002

Surman, R., McLaughlin, G. C., \& Hix, W. R. 2006, ApJ, 643, 1057

Tagliaferri, G., et al. 2005, Nature, 436, 985

Thompson, T. A., Chang, P., \& Quataert, E. 2004, ApJ, 611, 380

Thompson, T. A., Quataert, E., \& Burrows, A. 2005, ApJ, 620, 861

Timmes, F. X., \& Swesty, F. D. 2000, ApJS, 126, 501

van Marle, A. J., Owocki, S. P., \& Shaviv, N. J. 2008, MNRAS, 389, 1353

Vaughan, S., et al. 2006, ApJ, 638, 920

Wang, P., Abel, T., \& Zhang, W. 2008, ApJS, 176, 467

Wheeler, J. C., Yi, I., Höflich, P., \& Wang, L. 2000, ApJ, 537, 810

Woosley, S. E. 1993, ApJ, 405, 273

Woosley, S. E., \& Bloom, J. S. 2006, ARA\&A, 44, 507

Woosley, S. E., \& Heger, A. 2006, ApJ, 637, 914

Zhang, B., Fan, Y. Z., Dyks, J., Kobayashi, S., Mészáros, P., Burrows, D. N., Nousek, J. A., \& Gehrels, N. 2006, ApJ, 642, 354

Zhang, W., \& MacFadyen, A. I. 2006, ApJS, 164, 255

Zhang, B., \& Mészáros, P. 2001, ApJ, 552, L35

Zhang, W., Woosley, S. E., \& Heger, A. 2004, ApJ, 608, 365

Zhang, W., Woosley, S. E., \& Heger, A. 2008, ApJ, 679, 639

Zhang, W., Woosley, S. E., \& MacFadyen, A. I. 2003, ApJ, 586, 356

Zimmerman, E. R., Narayan, R., McClintock, J. E., \& Miller, J. M. 2005, ApJ, 618,832 